\title{
High resolution physical map of porcine chromosome 7 QTL region and comparative mapping of this region among vertebrate genomes
}

\author{
Julie Demars1, Juliette Riquet ${ }^{1}$, Katia Feve1, Mathieu Gautier², \\ Mireille Morisson ${ }^{1}$, Olivier Demeure ${ }^{3}$, Christine Renard ${ }^{4}$, Patrick Chardon ${ }^{4}$ \\ and Denis Milan*1
}

\begin{abstract}
Address: ${ }^{1}$ Laboratoire de Génétique Cellulaire, INRA, BP52627, 31326 Castanet-Tolosan, France, ${ }^{2}$ Laboratoire de Génétique Biochimique et de Cytogénétique, INRA, 78352 Jouy en Josas, France, ${ }^{3}$ Laboratoire de Génétique animale, INRA, 35042 Rennes, France and ${ }^{4}$ Laboratoire de Radiobiologie et d'Etude du Génome, INRA-CEA, 78352 Jouy en Josas, France

Email: Julie Demars - julie.demars@toulouse.inra.fr; Juliette Riquet - juliette.riquet@toulouse.inra.fr; Katia Feve - katia.feve@toulouse.inra.fr; Mathieu Gautier - mathieu.gautier@jouy.inra.fr; Mireille Morisson - mireille.morisson@toulouse.inra.fr;

Olivier Demeure - demeure@roazhon.inra.fr; Christine Renard - crenard@jouy.inra.fr; Patrick Chardon - Patrick.chardon@jouy.inra.fr;

Denis Milan* - denis.milan@toulouse.inra.fr

* Corresponding author
\end{abstract}

Published: 24 January 2006

BMC Genomics2006, 7:13 doi:10.1186/147|-2164-7-13
Received: 02 August 2005

Accepted: 24 January 2006

This article is available from: http://www.biomedcentral.com/I47I-2/64/7//3

(c) 2006Demars et al; licensee BioMed Central Ltd.

This is an Open Access article distributed under the terms of the Creative Commons Attribution License (http://creativecommons.org/licenses/by/2.0), which permits unrestricted use, distribution, and reproduction in any medium, provided the original work is properly cited.

\begin{abstract}
Background: On porcine chromosome 7, the region surrounding the Major Histocompatibility Complex (MHC) contains several Quantitative Trait Loci (QTL) influencing many traits including growth, back fat thickness and carcass composition. Previous studies highlighted that a fragment of $\sim 3.7 \mathrm{Mb}$ is located within the Swine Leucocyte Antigen (SLA) complex. Internal rearrangements of this fragment were suggested, and partial contigs had been built, but further characterization of this region and identification of all human chromosomal fragments orthologous to this porcine fragment had to be carried out.

Results: A whole physical map of the region was constructed by integrating Radiation Hybrid (RH) mapping, BAC fingerprinting data of the INRA BAC library and anchoring BAC end sequences on the human genome. 17 genes and 2 reference microsatellites were ordered on the high resolution IMNPRH2 ${ }_{12000 \text { rad }}$ Radiation Hybrid panel. A 1000:I framework map covering $550 \mathrm{CR}_{12000}$ was established and a complete contig of the region was developed. New micro rearrangements were highlighted between the porcine and human genomes. A bovine $\mathrm{RH}$ map was also developed in this region by mapping 16 genes. Comparison of the organization of this region in pig, cattle, human, mouse, dog and chicken genomes revealed that I) the translocation of the fragment described previously is observed only on the bovine and porcine genomes and 2) the new internal micro rearrangements are specific of the porcine genome.

Conclusion: We estimate that the region contains several rearrangements and covers $5.2 \mathrm{Mb}$ of the porcine genome. The study of this complete BAC contig showed that human chromosomal fragments homologs of this heavily rearranged QTL region are all located in the region of HSA6 that surrounds the centromere. This work allows us to define a list of all candidate genes that could explain these QTL effects.
\end{abstract}




\section{Background}

Chromosome 7 contains a region of the porcine genome in which the highest number of quantitative trait loci (QTL) have been mapped, affecting most notably growth, fat deposition and carcass composition [1-5]. Among all QTL detected on this chromosome, many have been mapped in an interval spanning the SLA complex in position 7p12-q12. Development of a high resolution porcine gene map and comparison with the human map is a major step towards the identification of the genes responsible for QTL effects. The development of two radiation hybrid panels IMpRH and IMNpRH2 constructed after irradiation of porcine cells at 7.000 and 12.000 rads [6,7] provides tools of choice for such comparative mapping studies at 2 complementary resolutions : 1) one breakage could be observed on at least one hybrid of the panel for markers at an average distance of $>50$ and $20 \mathrm{~Kb}, 2$ ) the order of a set of markers can be unambiguously determined, provided that the distance between adjacent markers range from 0.25 to $1.5 \mathrm{Mb}$ on IMpRH panel and from 100 to $600 \mathrm{~Kb}$ on IMNpRH2 panel [8].

At first glance, the chromosomal region in which QTL have been mapped on SSC7 seemed identical to the p arm of HSA6 $[9,10]$. A more detailed study showed that a fragment of $3.7 \mathrm{Mb}$ found at a pericentromeric location on HSA6 is situated at $23 \mathrm{Mb}$ from the expected location on the porcine genome, precisely in the region of interest $[11,12]$. This fragment was defined by three genes (FLJ10775, BMP5 and BAG2) located close to the centromere at $56 \mathrm{Mb}$ on HSA6p12.1-6p11.2 and mapped on SSC7q11 close to RAB2L and DAXX genes localized at 33 Mb on HSA6p21.32. A preliminary physical map of this region was proposed by Barbosa [12], but 4 limited BAC contigs were built in this rearranged region. However, it failed to establish a complete comparative map of this region and to ensure that no additional fragments found elsewhere on the human genome are located in the porcine QTL region.

We now report the building of a complete BAC map covering the whole region. This was achieved by taking advantage of the fingerprinting of a $5.5 \times$ BAC library (Chardon et al, in preparation), undertaken to establish primary BAC contigs. We monitored the assembly of the complete contig by mapping genes and markers on the high resolution IMNpRH2 12000 panel, as previously done in the Halothane region [8]. Sequence comparison of BAC ends from this contig with the human sequence allowed us to identify 4 BAC containing the four synteny breaking points detected in this region. A local bovine RH map was also established in this region to determine if the rearrangements observed on the pig genome are specific of suines or if they occurred in an ancestral species. A multispecies comparison of this region was finally performed.

\section{Results \\ Development of a preliminary porcine gene map}

To study the complete rearrangements that occurred in this region of an estimated $3.7 \mathrm{Mb}$ and containing the 3 genes LANO (previously called FLJ10775), BMP5 and BAG2 [11,12], it was necessary to: 1$)$ define the chromosomal blocks arranged differently between HSA6 pericentromeric region and SSC7; 2) determine if additional human fragments moved in this porcine region. In the first approach, we increased the density of genes mapped in this region of the IMNpRH2 $2_{12000}$ panel [7]. Using the ICCARE browser [13], we identified genes for which porcine EST were available. We chose 5 genes located on HSA6p21.32 and 5 genes located on HSA6p12.1-6p11.2: COL11A2 (33.24 Mb), HSD17B8 (33.28 Mb), RING1 (33.29 Mb), VPS52 (33.33 Mb), RPS18 (33.35 Mb), GCLC (53.47 Mb), TINAG (54.28 Mb), HCRTR2 (55.15 Mb), COL21A1 (56.03 Mb) and DST (56.43 Mb). No gene was selected below PRIM2A since no porcine sequence was available for the few genes located in this region very close to the centromere of HSA6. When possible, primers were developed in the 3' UTR sequence of selected genes (Table 1 ). For GCLC only, we had to choose primers in two consecutive exons framing an intron. Markers developed for these 10 genes, as well as markers previously developed [11,12] for RXRb (33.27 Mb), ELOVL5 (53.28 Mb) and PRIM2A (57.29 Mb), were analyzed on the 90 clones of IMNpRH $22_{12000}$ panel. In addition to markers produced in the frame of this project, we included in the analysis markers already mapped in this region on IMPRH and IMNpRH2 panels, in particular HKE4 (33.28 Mb), RAB2L (33.37 Mb), LANO (53.77 Mb) (called previously FLJ10775),BMP5 (55.73 Mb) and BAG2 (57.15 Mb) $[11,12]$. An average retention frequency of $40 \%$ was observed, ranging from $27 \%$ for PRIM2A to $55 \%$ for RAB2L and HKE4. A draft 1000:1 framework map was then built with Carthagene software [14], using a stepwise locus adding strategy (data not shown). The robustness of the map was tested by comparison of the likelihood of the reference map to the likelihood of maps derived from the reference map by all possible permutations in a window of 6 markers and by inversion of parts of the map using the simulated annealing algorithm. The 6 additional genes (COL11A2, RXRb, RING1, PRIM2A, COL21A1 and RPS18) were mapped at their most likely location relatively to markers of this preliminary framework map, considering the distance between framework markers as fixed.

To compare the order of genes between SSC7 and HSA6, we determined the location of these genes on the NCBI Build 35 assembly of the human genome available at UCSC [15]. We confirmed the insertion in pig of a large fragment between RING1 and VPS52 genes (fragments 1 and 2), which are only $40 \mathrm{~Kb}$ apart on the human genome (Figure 1A). The fragment found in pig between these two 


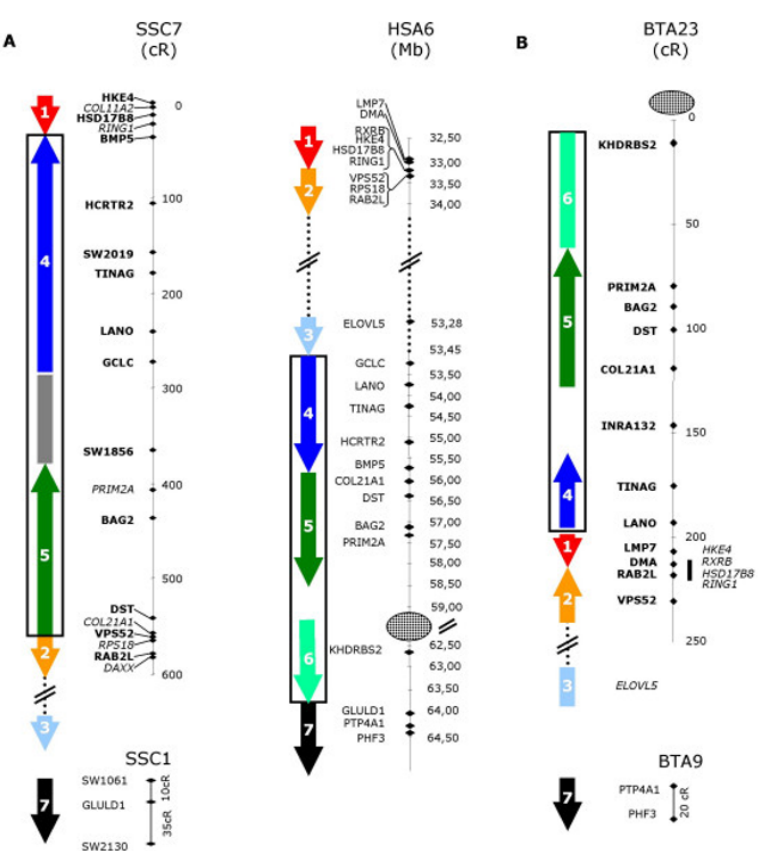

Figure I

Gene based comparative map of SSC $7 \mathrm{ql} .2$ and BTA $23 \mathrm{q} I \mathrm{I}$ radiation hybrid maps and HSA $6 \mathrm{p} 2$ I.32/HSA 6p I 2. I-q I I 2 physical map. A- SSC 7qI.2 radiation hybrid map was build using IMNpRH2 ${ }_{12000}$ developed by Yerle et al [7]. B- BTA 23q I I radiation hybrid map was build using RH5000 panels developed by Williams et al [22]. The human physical map corresponds to NCBI build 35 produced by the International Human Genome Sequencing Consortium. Markers in bold on the $\mathrm{RH}$ map belong to the framework map, whereas markers in italic are mapped at their most likely location considering as fixed the distance between framework markers. The centromere is represented by a circle. Arrows indicate blocks of gene whose order is conserved among the three species. Hatched box on the SSC7 $\mathrm{RH}$ map represents a fragment where no gene mapping information is available.

genes is located on human genome close to the centromere on the HSA6p arm. The mapping of these genes on the porcine RH map allowed us to distinguish two main conserved fragments: one fragment containing BMP5 HCTR2 - Sw2019 - TINAG - LANO - GCLC (fragment 4), and a second fragment containing DST, BAG2 and PRIM2A (fragment 5). The order of genes is conserved inside each fragment; however, the 2 blocks are oppositely oriented in the porcine and human genomes. A large distance is observed between GCLC and BAG2, suggesting that another fragment is inserted between them, possibly a fragment called "6" in the human genome. This additional porcine fragment is particularly interesting because it contains Sw1856, a marker mapped at the most likely location of the QTL of interest. At that step, it was not possible to determine whether the rearranged fragment includes a short fragment located on the q arm of HSA6. The first gene found on HSA6q arm also mapped on the porcine genome, being FLJ13159 located on SSC1 [11].

\section{Bac contig construction}

In order to confirm the rearrangements previously observed between HSA6 (6p21.32 and 6p12.1-11.2) and SSC7 (7q11), we built a complete BAC contig of this region. As in previous studies, an almost complete BAC contig of the SLA region has been developed [12], but a gap remained to be filled precisely in this region that was very different between the human and pig genomes. We screened by PCR the INRA BAC library [16] using primers defined for microsatellites Sw2019 and Sw1856, and for the 10 following genes RING1, HSD17B8, BMP5, HCRTR2, TINAG, LANO, GCLC, PRIM2A, BAG2 and DST. For each of these 10 genes or 2 markers (Sw2019 and Sw1856), we identified one positive BAC (Figure 2). To accelerate the establishment of a complete BAC contig, we took advantage of the availability of preliminary BAC contigs built after the fingerprinting of $72190 \mathrm{BAC}$ of the INRA library (Chardon et al, in preparation). It appeared that 8 of these 12 BAC were mapped in 8 different INRA contigs (contigs 5174, 1178, 5990, 3302, 2270, 137, 236 and 1421), containing from 3 to $21 \mathrm{BAC}$ (Figure 2).

In the frame of the INRA physical mapping project, 35 000 INRA BAC regularly dispersed on the INRA contigs had been selected. The sequence of their extremities were determined at CNS (Evry, France) and compared with the human sequence (Chardon et al, in preparation). Analysis of 108 porcine BES anchored on the human region between 33 and $34 \mathrm{Mb}$, or between 53 and $64 \mathrm{Mb}$, allowed us to identify 55 BAC located on the pig genome in the chosen region. Among these BAC, 39 belonged to one of the 8 INRA contigs already identified. 8 additional BAC allowed us to identify 3 others INRA contigs (contigs 5488,6382 and 7444) located in this same region, and the last 8 clones correspond to BAC that had not been fingerprinted.

To fill several gaps, the INRA BAC library was screened with markers derived from BES, and 12 additional INRA BAC (bI0149A04, bI0474F10, bI0403D01, bI0842H05, bI0323A08, bI0159D05, bI0438F07, bI1044F02, bI0135F11, bI0311E12, bI0105B03 and bI0032D02) were recruited. For BAC of interest whose BES had not been sequenced by the CNS, we sequenced the extremities in order to get on with the construction of the contig; these sequences were submitted to EMBL Nucleotide Sequence Database (AM156864 to AM156903). To fill the remaining gaps, we selected 7 additional BAC (bE0164A10, RPCI44_0470A18, bE0047O02, 
Table I: Porcine markers developed for RH mapping and BAC contig construction

\begin{tabular}{|c|c|c|c|c|c|c|c|}
\hline STS-EST & Sequence up & Sequence on & $\begin{array}{l}\text { Size of } \\
\text { PCR } \\
\text { fragment }\end{array}$ & $\begin{array}{c}\text { \% Retention } \\
\text { on } \\
\text { IMNpRH2 }\end{array}$ & $\begin{array}{l}\text { Position } \\
\text { (cR) }\end{array}$ & $\begin{array}{l}\text { No INRA } \\
\text { Contig }\end{array}$ & $\begin{array}{c}\text { Human } \\
\text { Location } \\
\text { (Mb) }\end{array}$ \\
\hline \multicolumn{8}{|c|}{ Arrow number I on figure 2} \\
\hline bl0I49A04F & $\begin{array}{l}\text { CTCTCCTTCAAAG } \\
\text { GCCTCCT }\end{array}$ & $\begin{array}{l}\text { ATGCCTCAGGCGA } \\
\text { TCTAATG }\end{array}$ & 233 & & & & 33.03 \\
\hline COLIIA2 & $\begin{array}{l}\text { CTCCCACCTGGAG } \\
\text { ССTGT }\end{array}$ & $\begin{array}{l}\text { CAGTGTCCTTTCTC } \\
\text { TTCTCTTCC }\end{array}$ & 154 & $48 \%$ & & & 33.24 \\
\hline$R X R B$ & $\begin{array}{l}\text { TCCAGAGTCCCCT } \\
\text { CTCACAC }\end{array}$ & $\begin{array}{l}\text { CCAAATACCACCT } \\
\text { CСАССAC }\end{array}$ & 247 & $41 \%$ & & & 33.27 \\
\hline HKE4 & $\begin{array}{l}\text { CCGCTCTCTGCTC } \\
\text { CAGAT }\end{array}$ & $\begin{array}{l}\text { AAGAAAGGCGACA } \\
\text { ATTCCAC }\end{array}$ & 487 & $42 \%$ & 0 & & 33.28 \\
\hline HSDI7B8 & $\begin{array}{l}\text { CTCAAGGACCCTG } \\
\text { GACTCTG }\end{array}$ & $\begin{array}{l}\text { GGAATTTATTAGG } \\
\text { GTCACAAGCA }\end{array}$ & 186 & $45 \%$ & 15 & & 33.28 \\
\hline bl0392F02R & $\begin{array}{l}\text { AAGATGCCCTTGA } \\
\text { GCTGCTA }\end{array}$ & $\begin{array}{l}\text { GCAGCAGCACATT } \\
\text { GAGAGAA }\end{array}$ & 136 & & & & \\
\hline RINGI & $\begin{array}{l}\text { GCCACTTGTTCTC } \\
\text { ATTTGTGTC }\end{array}$ & $\begin{array}{l}\text { ATCCAAAGTGACC } \\
\text { CCACAAG }\end{array}$ & 150 & $48 \%$ & & & 33.28 \\
\hline \multicolumn{8}{|c|}{ Arrow number 4 on figure 2} \\
\hline bl0I49A04R & $\begin{array}{l}\text { CTTGGATCAAACC } \\
\text { CACATCC }\end{array}$ & $\begin{array}{l}\text { CGGCGTGTAGTGA } \\
\text { TTGCTTA }\end{array}$ & 282 & & & & 55.93 \\
\hline bl0248H04R & $\begin{array}{l}\text { AGGGAATGACAAC } \\
\text { AGCAACC }\end{array}$ & $\begin{array}{l}\text { TGGAGCAGATTTA } \\
\text { GGATTTGG }\end{array}$ & 163 & & & 5174 & 55.80 \\
\hline bI0I23E02R & $\begin{array}{l}\text { CCGAGTGGAATCA } \\
\text { TATGCTCTT }\end{array}$ & $\begin{array}{l}\text { TCCTCGACGAAGT } \\
\text { TCTCCAT }\end{array}$ & 153 & & & 5174 & 55.78 \\
\hline bE276E04SP6 & $\begin{array}{l}\text { GTAGGGTTAGCCT } \\
\text { GCTGTGG }\end{array}$ & $\begin{array}{l}\text { TGTCGAAACTTGG } \\
\text { CСTCTCT }\end{array}$ & 127 & & & & 55.77 \\
\hline BMP5 & $\begin{array}{l}\text { ACAGCAAAAAGCA } \\
\text { AAGACCA }\end{array}$ & $\begin{array}{l}\text { GTAAGTAGGTTGT } \\
\text { CAGGCTTGC }\end{array}$ & 230 & $41 \%$ & 32 & & 55.73 \\
\hline bl0895FI2R & $\begin{array}{l}\text { GGCAAAGCAATTC } \\
\text { CACAGAT }\end{array}$ & $\begin{array}{l}\text { CCGAAACCTCATG } \\
\text { GTTCCTA }\end{array}$ & 198 & $33 \%$ & 59 & 5174 & 55.71 \\
\hline bl0I 23E02F & $\begin{array}{l}\text { GAGACCCAGAAAC } \\
\text { CAACCAA }\end{array}$ & $\begin{array}{l}\text { GCAGGCTTGTCTC } \\
\text { CAATGAC }\end{array}$ & 249 & & & 5174 & 55.67 \\
\hline bl0474FIOF & $\begin{array}{l}\text { CAAGTGAAGCAAA } \\
\text { TCCACCA }\end{array}$ & $\begin{array}{l}\text { ACTCCTGCACTGG } \\
\text { CCATATT }\end{array}$ & 232 & & & & \\
\hline bl0474FIOR & $\begin{array}{l}\text { CATGCTTGCTAAC } \\
\text { ACCAGGA }\end{array}$ & $\begin{array}{l}\text { AGTGGCTCTCAGC } \\
\text { CTCAAAA }\end{array}$ & 176 & & & & \\
\hline bI0I87D03R & $\begin{array}{l}\text { AGAAACACCAGCC } \\
\text { TCCTCAA }\end{array}$ & $\begin{array}{l}\text { CCACAGCACAGTG } \\
\text { CAGAAAT }\end{array}$ & 256 & $43 \%$ & 82 & 5488 & 55.51 \\
\hline bl0I87D03F & $\begin{array}{l}\text { CGACACCCACAAA } \\
\text { GGAAAGT }\end{array}$ & $\begin{array}{l}\text { GTGATGCATTTTG } \\
\text { CCACATC }\end{array}$ & 169 & & & 5488 & \\
\hline bl0773C07R & $\begin{array}{l}\text { AGAAAGGTGGGGA } \\
\text { GAGCATT }\end{array}$ & $\begin{array}{l}\text { GCACTGGAGCACA } \\
\text { GCAAATA }\end{array}$ & 132 & & & & 55.36 \\
\hline bl0I36E09F & $\begin{array}{l}\text { CTGCTTGTGGGGA } \\
\text { TACCATT }\end{array}$ & $\begin{array}{l}\text { TCCTCACCTGATT } \\
\text { CGCTACA }\end{array}$ & 158 & $35 \%$ & 89 & 5488 & 55.30 \\
\hline bl0773C07F & $\begin{array}{l}\text { GGCATGTTGAAAG } \\
\text { CCAAGAT }\end{array}$ & $\begin{array}{l}\text { TTTGGCTTAAGGG } \\
\text { AATGTCG }\end{array}$ & 213 & & & & \\
\hline bE0I64AIOSP6 & $\begin{array}{l}\text { GCACCTGGACTGG } \\
\text { AGGAATA }\end{array}$ & $\begin{array}{l}\text { AGGGCCTAGGTCC } \\
\text { ACTCAAT }\end{array}$ & 115 & & & & 55.23 \\
\hline HCRTR2 & $\begin{array}{l}\text { TGTGTCGGTGTCT } \\
\text { GTGTCTG }\end{array}$ & $\begin{array}{l}\text { CGATCCAGATGAT } \\
\text { GACGATG }\end{array}$ & 131 & $44 \%$ & 101 & & 55.15 \\
\hline bE0I64AI0T7 & $\begin{array}{l}\text { AATCTTTGGGCAG } \\
\text { TGAGTCG }\end{array}$ & $\begin{array}{l}\text { CATGGAATCACCG } \\
\text { AAATGTG }\end{array}$ & 230 & & & & 55.01 \\
\hline bl0759D02R & $\begin{array}{l}\text { TTTTAACGCAATCT } \\
\text { GCTTGG }\end{array}$ & $\begin{array}{l}\text { CAGCTCCCTCATT } \\
\text { TTGAAGC }\end{array}$ & 151 & $45 \%$ & 121 & 6382 & 54.90 \\
\hline bl0699BIOR & $\begin{array}{l}\text { TGTTGGTTTGGGT } \\
\text { GTTGAGA }\end{array}$ & $\begin{array}{l}\text { TCTTGAGGCAGTG } \\
\text { TGCATTC }\end{array}$ & 262 & & & 6382 & \\
\hline bE0047O02T7 & $\begin{array}{l}\text { CTCACCTCCATCC } \\
\text { АСАACCT }\end{array}$ & $\begin{array}{l}\text { GCTGGTGCCAAGA } \\
\text { TCCTAAG }\end{array}$ & 235 & & & & 54.83 \\
\hline $\begin{array}{l}\text { RPCl44_0470A } \\
18 \mathrm{~F}\end{array}$ & $\begin{array}{l}\text { CCTAAGTGCCATT } \\
\text { СТССТССT }\end{array}$ & $\begin{array}{l}\text { AAAGCAGGCCACT } \\
\text { CGAAGTA }\end{array}$ & 241 & & & & 54.82 \\
\hline bIOI3IFOIR & $\begin{array}{l}\text { CACCACACACAGC } \\
\text { AACATCA }\end{array}$ & $\begin{array}{l}\text { TCAGGAGCTCCCA } \\
\text { GGAATAA }\end{array}$ & 103 & $41 \%$ & 138 & 1178 & \\
\hline
\end{tabular}


Table I: Porcine markers developed for RH mapping and BAC contig construction (Continued)

\begin{tabular}{|c|c|c|c|c|c|c|c|}
\hline bI0I3IFOIF & $\begin{array}{l}\text { CCTCTCTGGTCTC } \\
\text { TGCATCC }\end{array}$ & $\begin{array}{l}\text { CTCCTGTCTTCCA } \\
\text { GCTCCTG }\end{array}$ & 157 & & & 1178 & 54.78 \\
\hline SW2019 & $\begin{array}{l}\text { ATGATGCGAACCT } \\
\text { GGAACTC }\end{array}$ & $\begin{array}{l}\text { TATGTGTAACTTGG } \\
\text { TCCCATGC }\end{array}$ & & $30 \%$ & 153 & & \\
\hline bl0444G09F & $\begin{array}{l}\text { GGCAATCATTTCC } \\
\text { ACCTTGT }\end{array}$ & $\begin{array}{l}\text { AGAACTGGAGGCA } \\
\text { GTGGAGA }\end{array}$ & 231 & & & 1178 & \\
\hline bl0704C03F & $\begin{array}{l}\text { CGGCCCTATTTTTC } \\
\text { ACTTCA }\end{array}$ & $\begin{array}{l}\text { CTCCCTGGTGCCA } \\
\text { AATAGAA }\end{array}$ & 172 & & & 1178 & 54.55 \\
\hline bl0295F03F & $\begin{array}{l}\text { CCCACCCAAGGTT } \\
\text { GACTAGA }\end{array}$ & $\begin{array}{l}\text { GGAACTCCCAACG } \\
\text { TGAACTC }\end{array}$ & 129 & & & 5990 & 54.41 \\
\hline bl0704C03R & $\begin{array}{l}\text { AACCCAGGACCCA } \\
\text { AGGATAC }\end{array}$ & $\begin{array}{l}\text { ACTCTCСTTCCAC } \\
\text { AGCCAGA }\end{array}$ & 232 & $33 \%$ & & 1178 & 54.31 \\
\hline TINAG & $\begin{array}{l}\text { ATTCCTGGGGAAA } \\
\text { GTCATGG }\end{array}$ & $\begin{array}{l}\text { AAGGCATGACCTT } \\
\text { ATGGAAAT }\end{array}$ & 157 & $33 \%$ & 175 & & 54.28 \\
\hline bl0295F03R & $\begin{array}{l}\text { GTGGTGCAGAGGG } \\
\text { TTAAGGA }\end{array}$ & $\begin{array}{l}\text { TAGGGCTTTGCTT } \\
\text { GTCTCGT }\end{array}$ & 269 & $47 \%$ & & 5990 & \\
\hline bl07I6B06F & $\begin{array}{l}\text { TCCATTTGTTGGG } \\
\text { GGTTTTA }\end{array}$ & $\begin{array}{l}\text { ATAAGCCTCAGCC } \\
\text { TCCATGA }\end{array}$ & 177 & & & 5990 & 54.19 \\
\hline bl0355A07F & $\begin{array}{l}\text { GGCCAGATGGAAA } \\
\text { TCTTTGA }\end{array}$ & $\begin{array}{l}\text { CCAGCGGATGTGT } \\
\text { CCTATTT }\end{array}$ & 252 & $29 \%$ & 196 & 3302 & 54.17 \\
\hline bI06I5FIOR & $\begin{array}{l}\text { TCTGTTTCCCATTT } \\
\text { GCACCT }\end{array}$ & $\begin{array}{l}\text { CCACTGCAGTCAA } \\
\text { GGTCTCA }\end{array}$ & 186 & & & 3302 & 0.00 \\
\hline bl0872D06F & $\begin{array}{l}\text { TCATGCTGGCAAA } \\
\text { TGGTAAA }\end{array}$ & $\begin{array}{l}\text { ATTTCTGGCAGAG } \\
\text { CGGATAA }\end{array}$ & 116 & $33 \%$ & & 3302 & 53.78 \\
\hline bl0438F07R & $\begin{array}{l}\text { CGGGTGGAAGGAG } \\
\text { ATCACTA }\end{array}$ & $\begin{array}{l}\text { TTCAAAGAAGGCA } \\
\text { TGGTTCC }\end{array}$ & 244 & & & & 53.77 \\
\hline LANO & $\begin{array}{l}\text { GGGATGCTGAATG } \\
\text { TGGTATTT }\end{array}$ & $\begin{array}{l}\text { TTTTCTCTTTTTGT } \\
\text { GCTTTTCTG }\end{array}$ & 287 & $38 \%$ & 235 & & 53.77 \\
\hline bll 044F02R & $\begin{array}{l}\text { TACATGCACAGTT } \\
\text { GGGGAAA }\end{array}$ & $\begin{array}{l}\text { ACCCCTTTGGTGG } \\
\text { TGTTGTA }\end{array}$ & 144 & & & & \\
\hline bl0547G I 2F & $\begin{array}{l}\text { ATCTCTGAGCAGG } \\
\text { TCGCAGT }\end{array}$ & $\begin{array}{l}\text { ATCTTCCСTGATC } \\
\text { СССАСТT }\end{array}$ & 188 & & & 2270 & \\
\hline bl0547G I2R & $\begin{array}{l}\text { TGTCAGGGTGTCG } \\
\text { GTTGTAG }\end{array}$ & $\begin{array}{l}\text { GTCCTAACCCATG } \\
\text { CAGCAGT }\end{array}$ & 174 & & & 2270 & \\
\hline bl0285E03R & $\begin{array}{l}\text { AATTCCTCCGCTC } \\
\text { ACTCTGA }\end{array}$ & $\begin{array}{l}\text { TCAGGAGGGGAAG } \\
\text { CTGACTA }\end{array}$ & 174 & & & 2270 & \\
\hline GCLC & $\begin{array}{l}\text { ACCATCCTACCCT } \\
\text { TTGGAGA }\end{array}$ & $\begin{array}{l}\text { TTTCCCCCAGTAA } \\
\text { AGACGTG }\end{array}$ & 150 & $37 \%$ & 268 & & 53.47 \\
\hline bl0879H07F & $\begin{array}{l}\text { CTGGACGTGGAGT } \\
\text { CTGGTTT }\end{array}$ & $\begin{array}{l}\text { TTGCCCTTGATGC } \\
\text { TAAATCC }\end{array}$ & 300 & $38 \%$ & 324 & 2270 & \\
\hline \multicolumn{8}{|c|}{ Arrow number 6 on figure 2} \\
\hline bE25IO22T7 & $\begin{array}{l}\text { TGGCAGCTTGACA } \\
\text { TCAGTTC }\end{array}$ & $\begin{array}{l}\text { AATGTGCCAGAGG } \\
\text { GTTTGAG }\end{array}$ & 210 & & & & 62.48 \\
\hline bl0879H07R & $\begin{array}{l}\text { GAGCCTGTTAGCG } \\
\text { CATTTCT }\end{array}$ & $\begin{array}{l}\text { ATGCCAAACCATC } \\
\text { TGTCCTC }\end{array}$ & 189 & & & 2270 & 62.51 \\
\hline bT79E I8SP6 & $\begin{array}{l}\text { TGTGGTATGAACC } \\
\text { CAGGACA }\end{array}$ & $\begin{array}{l}\text { AAGGGGATTTTCC } \\
\text { CCATGTA }\end{array}$ & 211 & & & & 62.60 \\
\hline bE235D|4SP6 & $\begin{array}{l}\text { TCGGAAGATTGCC } \\
\text { TATTTGG }\end{array}$ & $\begin{array}{l}\text { TGAAGATGACTGC } \\
\text { TGCCTTG }\end{array}$ & 192 & & & & 62.67 \\
\hline bE25IO22SP6 & $\begin{array}{l}\text { ATCTGGCCCCTGT } \\
\text { TCTCTTT }\end{array}$ & $\begin{array}{l}\text { GAAAATTTCTCTCC } \\
\text { ССТGTGC }\end{array}$ & 232 & & & & 62.73 \\
\hline bl0052D02F & $\begin{array}{l}\text { ACAGCATTATGCC } \\
\text { GATTTCT }\end{array}$ & $\begin{array}{l}\text { CCTACAAGAACCT } \\
\text { TACAGCATTAGA }\end{array}$ & 100 & & & 137 & 62.78 \\
\hline bl0052D02R & $\begin{array}{l}\text { TGGCTTTATAATTA } \\
\text { TGTGCTAATGAC }\end{array}$ & $\begin{array}{l}\text { CCTACATGGGAAA } \\
\text { AGAATTTGAA }\end{array}$ & 169 & $27 \%$ & & 137 & \\
\hline bI0I37BI2F & $\begin{array}{l}\text { GCATGGAGTTTGG } \\
\text { TGTTGTG }\end{array}$ & $\begin{array}{l}\text { TTAGGTGAAAGGC } \\
\text { CTGATGG }\end{array}$ & 165 & & & 137 & \\
\hline bI0I37BI2R & $\begin{array}{l}\text { CCAAACCCAAACT } \\
\text { CCCTGTA }\end{array}$ & $\begin{array}{l}\text { TCAGCAACATGGA } \\
\text { TGCAACT }\end{array}$ & 218 & & & 137 & \\
\hline bl0666EOIF & $\begin{array}{l}\text { GCATGCAAAGGCA } \\
\text { CTCAGTA }\end{array}$ & $\begin{array}{l}\text { CAAGAGCAAATGC } \\
\text { ATTCCAA }\end{array}$ & $17 \mid$ & $35 \%$ & & 236 & 62.91 \\
\hline bl0455FOIR & $\begin{array}{l}\text { CAAGAGCAAATGC } \\
\text { ATTCCAA }\end{array}$ & $\begin{array}{l}\text { GCATGCAAAGGCA } \\
\text { CTCAGTA }\end{array}$ & $17 \mid$ & $35 \%$ & & 137 & 62.91 \\
\hline bI04I5F05R & $\begin{array}{l}\text { TCTGAGCTGCATTT } \\
\text { GTGACC }\end{array}$ & $\begin{array}{l}\text { GCTGGTTCTGAAT } \\
\text { CCCCATA }\end{array}$ & 249 & & & 236 & 62.98 \\
\hline
\end{tabular}


Table I: Porcine markers developed for RH mapping and BAC contig construction (Continued)

\begin{tabular}{|c|c|c|c|c|c|c|c|}
\hline \\
\hline bl0688H06R & $\begin{array}{l}\text { GCATCTTTGCATG } \\
\text { AGCCATA }\end{array}$ & $\begin{array}{l}\text { CATTGGCTAGAGA } \\
\text { GAAGAGAAACA }\end{array}$ & 189 & & & 236 & 63.02 \\
\hline bl0666E0IR & $\begin{array}{l}\text { CCTCCGGACACTA } \\
\text { CTTCCAA }\end{array}$ & $\begin{array}{l}\text { CAAAGACACACGC } \\
\text { TGAGGAA }\end{array}$ & 221 & & & 236 & 63.05 \\
\hline bl0374A03R & $\begin{array}{l}\text { CAAATGCCAGGTG } \\
\text { GTGAAAT }\end{array}$ & $\begin{array}{l}\text { CCATTTTCTGAGAA } \\
\text { GGGGTCT }\end{array}$ & 192 & & & 236 & \\
\hline bl04I5F05F & $\begin{array}{l}\text { AGCAATGGAAAAG } \\
\text { CAACTGG }\end{array}$ & $\begin{array}{l}\text { CGGGAACTCCTCT } \\
\text { GCTGTT }\end{array}$ & 212 & & & 236 & \\
\hline bl0688H06F & $\begin{array}{l}\text { ATGTTTGCACATTA } \\
\text { TGCCTTT }\end{array}$ & $\begin{array}{l}\text { ACTGCAGGCCATT } \\
\text { CTGACTT }\end{array}$ & 234 & & & 236 & 63.67 \\
\hline SWI 856 & $\begin{array}{l}\text { TTGTATGGTATCCT } \\
\text { GTGATGCC }\end{array}$ & $\begin{array}{l}\text { TCATTCCAAACAC } \\
\text { ACAGAGTCC }\end{array}$ & & $42 \%$ & 361 & & \\
\hline bl0586CIIR & $\begin{array}{l}\text { GCTGAGTTGATGC } \\
\text { TTGACCA }\end{array}$ & $\begin{array}{l}\text { CCCACTTGGGCAA } \\
\text { AGTAAAG }\end{array}$ & 193 & & & 236 & 63.70 \\
\hline bl0222C06F & $\begin{array}{l}\text { TCCCGACTACAGA } \\
\text { CCAAAGG }\end{array}$ & $\begin{array}{l}\text { GCATTCGATAGCA } \\
\text { ATTTGGAA }\end{array}$ & 199 & & & 236 & 63.70 \\
\hline bl0374A03F & $\begin{array}{l}\text { TGGGTGCAAAAAG } \\
\text { TTTCCAG }\end{array}$ & $\begin{array}{l}\text { AGAGCGACCCAAG } \\
\text { AAAGTGA }\end{array}$ & $|5|$ & & & 236 & \\
\hline bl0834FIOR & $\begin{array}{l}\text { AGGCTCCAGGCAA } \\
\text { GAGAAG }\end{array}$ & $\begin{array}{l}\text { GAGAGGAGGTTTG } \\
\text { CCAGAGA }\end{array}$ & 247 & & & 236 & \\
\hline bl0492A07R & $\begin{array}{l}\text { GGCATTTGATGCT } \\
\text { GACACAC }\end{array}$ & $\begin{array}{l}\text { CATTCGGGGATAG } \\
\text { CGTTTTA }\end{array}$ & 153 & & & 236 & 63.70 \\
\hline bl0834FIOF & $\begin{array}{l}\text { AACTCTGCTTCCC } \\
\text { AGTGCAT }\end{array}$ & $\begin{array}{l}\text { TAGGCTGCAGGCC } \\
\text { TTTTCTA }\end{array}$ & 222 & & & 236 & \\
\hline bl0586Cl IF & $\begin{array}{l}\text { ACCTTGGACGAAG } \\
\text { TCССТCT }\end{array}$ & $\begin{array}{l}\text { GACATCCAGCATC } \\
\text { AGCTCAA }\end{array}$ & 207 & & & 236 & \\
\hline \multicolumn{8}{|c|}{ Arrow number 5 on figure 2} \\
\hline bl0223CIOR & $\begin{array}{l}\text { CTTGGGCAAGGCT } \\
\text { GATAGAC }\end{array}$ & $\begin{array}{l}\text { CATTCCATAGTCG } \\
\text { GGTGTTTTT }\end{array}$ & 168 & & & 236 & 58.03 \\
\hline bl0762HI2R & $\begin{array}{l}\text { TGGCCCTAAAAAG } \\
\text { ACCAAAA }\end{array}$ & $\begin{array}{l}\text { TGGACTGGGAAAC } \\
\text { AACTTCC }\end{array}$ & 176 & & & 236 & 57.78 \\
\hline bl0492A07F & $\begin{array}{l}\text { GGGCATCATCGTG } \\
\text { TTCTTTT }\end{array}$ & $\begin{array}{l}\text { GGCACAACTTTGA } \\
\text { CCTCATGT }\end{array}$ & 240 & & & 236 & 57.69 \\
\hline bl0392B02R & $\begin{array}{l}\text { GATGGCCTGGTGG } \\
\text { TTAAAGA }\end{array}$ & $\begin{array}{l}\text { AGTGAAAAATGCC } \\
\text { ACCAACC }\end{array}$ & 180 & & & 236 & 57.67 \\
\hline bl0223ClOF & $\begin{array}{l}\text { TGTTCCAAAGGAA } \\
\text { AATAGAACAAA }\end{array}$ & $\begin{array}{l}\text { AAAGCAGAAGTGA } \\
\text { CCCAGCTA }\end{array}$ & 227 & & & 236 & 57.65 \\
\hline bl0I86F03R & $\begin{array}{l}\text { GGTGCGGTCAAAA } \\
\text { ATGAAAA }\end{array}$ & $\begin{array}{l}\text { TAAAAATCCAGGG } \\
\text { CCACTCA }\end{array}$ & 187 & & & 236 & 57.58 \\
\hline $\begin{array}{l}\text { RPCl44_0334P2 } \\
\text { OR }\end{array}$ & $\begin{array}{l}\text { CCTCTGCCAGCCT } \\
\text { CATTTAC }\end{array}$ & $\begin{array}{l}\text { GCTTCAGGGAAGG } \\
\text { GGATTAG }\end{array}$ & 144 & & & & 57.56 \\
\hline bl0392B02F & $\begin{array}{l}\text { СTCCCTTGTGTCC } \\
\text { АСTTGCT }\end{array}$ & $\begin{array}{l}\text { AGAGCACCACCCT } \\
\text { TGAGAGA }\end{array}$ & 206 & $32 \%$ & & 236 & 57.55 \\
\hline bl0877E0IR & $\begin{array}{l}\text { CTСССССТССТСA } \\
\text { GCTAAAT }\end{array}$ & $\begin{array}{l}\text { TGAGGTTCACCGG } \\
\text { ATCTTTC }\end{array}$ & 195 & $34 \%$ & 412 & 7444 & 57.52 \\
\hline bI0877EOIF & $\begin{array}{l}\text { GGCCATTTGCAGT } \\
\text { GTCTTTT }\end{array}$ & $\begin{array}{l}\text { AAGCCTCAGGAAC } \\
\text { AATGCTG }\end{array}$ & 164 & & & 7444 & 57.39 \\
\hline $\begin{array}{l}\text { RPCl44_0334P2 } \\
\text { OF }\end{array}$ & $\begin{array}{l}\text { TCCCCСTTTGTTTC } \\
\text { TTCСТT }\end{array}$ & $\begin{array}{l}\text { CACGCAAATCAGA } \\
\text { AGATGGA }\end{array}$ & 195 & & & & 57.38 \\
\hline bl0297AIIR & $\begin{array}{l}\text { CAGAATGCCAAGA } \\
\text { GGGAGAG }\end{array}$ & $\begin{array}{l}\text { CAGAATGCCAAGA } \\
\text { GGGAGAG }\end{array}$ & 161 & & & 7444 & 57.36 \\
\hline PRIM2A & $\begin{array}{l}\text { CATAGCTTCGGAA } \\
\text { AGGAAGG }\end{array}$ & $\begin{array}{l}\text { CTTGGTGGATTGG } \\
\text { TGAGGAT }\end{array}$ & & & & & 57.29 \\
\hline bl0297AIIF & $\begin{array}{l}\text { CCACTGCCACTGA } \\
\text { CTCTTCA }\end{array}$ & $\begin{array}{l}\text { TCAATGCAATCCC } \\
\text { TGTCAAA }\end{array}$ & 266 & & 425 & 7444 & 0.00 \\
\hline bl0229FOIF & $\begin{array}{l}\text { CTGAGGGGAAGCC } \\
\text { TAGACAG }\end{array}$ & $\begin{array}{l}\text { CAGGAATACAGCC } \\
\text { CCTACCA }\end{array}$ & 227 & $41 \%$ & & $|42|$ & 57.12 \\
\hline BAG2 & $\begin{array}{l}\text { CGCCTGTTCTTCC } \\
\text { GAGGT }\end{array}$ & $\begin{array}{l}\text { GCATTTCTACGCC } \\
\text { ATTATTTCAAG }\end{array}$ & 255 & $39 \%$ & 432 & & 57.15 \\
\hline bl0229F0IR & $\begin{array}{l}\text { TGCCACGGCATAT } \\
\text { ATTTTCA }\end{array}$ & $\begin{array}{l}\text { CTACACCAGAGCC } \\
\text { ACAGCAA }\end{array}$ & 202 & & & $142 \mid$ & \\
\hline bl0192A07F & $\begin{array}{l}\text { CCGACTAGGATCC } \\
\text { ATGAGGA }\end{array}$ & $\begin{array}{l}\text { CCTTTTTAGGGCT } \\
\text { GCACTTG }\end{array}$ & 198 & & & $|42|$ & \\
\hline bl0192A07R & $\begin{array}{l}\text { ACCCACTGATCGA } \\
\text { CGTTAGG }\end{array}$ & $\begin{array}{l}\text { GTGGTTGGAATTT } \\
\text { GGCACTT }\end{array}$ & 209 & & & $|42|$ & 56.86 \\
\hline
\end{tabular}


Table I: Porcine markers developed for RH mapping and BAC contig construction (Continued)

\begin{tabular}{|c|c|c|c|c|c|c|c|}
\hline \\
\hline bI0783E0IR & $\begin{array}{l}\text { GAGGGCCTCTTAG } \\
\text { ATGCTGA }\end{array}$ & $\begin{array}{l}\text { CACCCATAAGGCT } \\
\text { TCСТCTG }\end{array}$ & 260 & $38 \%$ & 458 & $|42|$ & \\
\hline bl0697C09R & $\begin{array}{l}\text { TTATCACTGCTGTG } \\
\text { GCTTGG }\end{array}$ & $\begin{array}{l}\text { TCССТСССТАTTG } \\
\text { СТТССТT }\end{array}$ & 213 & $35 \%$ & 479 & & \\
\hline bl0697C09F & $\begin{array}{l}\text { ATGAGGCGAAACG } \\
\text { AAATGAC }\end{array}$ & $\begin{array}{l}\text { CAATGACTCGTGC } \\
\text { TGGTTTG }\end{array}$ & 191 & $25 \%$ & 507 & $142 \mid$ & 56.67 \\
\hline bI0I35FIIR & $\begin{array}{l}\text { CCCTCTGACAACC } \\
\text { ACCAGTT }\end{array}$ & $\begin{array}{l}\text { GCAACATGGAGGG } \\
\text { ACCTAGA }\end{array}$ & 163 & & & & 56.68 \\
\hline bl0I35FIIF & $\begin{array}{l}\text { TTGACCCTGTCGA } \\
\text { CTGCTTT }\end{array}$ & $\begin{array}{l}\text { CAACACAGTGGTT } \\
\text { GAACGTTTT }\end{array}$ & 185 & & & & \\
\hline bT220006SP6 & $\begin{array}{l}\text { TGAGAGGCCTCGT } \\
\text { GGATTAC }\end{array}$ & $\begin{array}{l}\text { TTGGAGGTGGCTC } \\
\text { AGAACTT }\end{array}$ & 155 & & & & 56.58 \\
\hline DST & $\begin{array}{l}\text { TGTCAGGTTTTCTT } \\
\text { TTGCTTGA }\end{array}$ & $\begin{array}{l}\text { GGGACACGTTTTA } \\
\text { TTTCATAGCTT }\end{array}$ & 106 & $32 \%$ & 538 & & 56.58 \\
\hline bl0I05B03F & $\begin{array}{l}\text { TCAGGGCACTTTC } \\
\text { TGCTGTA }\end{array}$ & $\begin{array}{l}\text { GCTGGGAGTTTGG } \\
\text { GGTTAGT }\end{array}$ & 183 & & & & \\
\hline bl0I05B03R & $\begin{array}{l}\text { TCAGTTGGAGCTG } \\
\text { AAAAACTCA }\end{array}$ & $\begin{array}{l}\text { AAACCACAAAAGC } \\
\text { CAAACCA }\end{array}$ & 226 & & & & 56.47 \\
\hline bT220006T7 & $\begin{array}{l}\text { GAATGGGAGGAGG } \\
\text { AAGTGGT }\end{array}$ & $\begin{array}{l}\text { CAGCTAAAGGACG } \\
\text { GACGAAC }\end{array}$ & 228 & & & & 56.44 \\
\hline bl0032D02F & $\begin{array}{l}\text { TCTGTGTACCACC } \\
\text { CCACTCA }\end{array}$ & $\begin{array}{l}\text { CCATGGCTGGTAT } \\
\text { TTGAACC }\end{array}$ & 257 & & & & 56.15 \\
\hline bl0032D02R & $\begin{array}{l}\text { AGGGGCCTGTTTA } \\
\text { CGTCTTT }\end{array}$ & $\begin{array}{l}\text { CACCTTGACGTCT } \\
\text { CATCAGC }\end{array}$ & 120 & & & & \\
\hline COL2IAI & $\begin{array}{l}\text { TGCATGCTTTCATT } \\
\text { TTCCAT }\end{array}$ & $\begin{array}{l}\text { TGTTCCTTAACAAC } \\
\text { GAAGCATT }\end{array}$ & 204 & $37 \%$ & & & 56.03 \\
\hline \multicolumn{8}{|c|}{ Arrow number 2 on figure 2} \\
\hline VPS52 & $\begin{array}{l}\text { CAAGAAGCACAAG } \\
\text { CCCAACT }\end{array}$ & $\begin{array}{l}\text { GGGACCCAGCTTA } \\
\text { TCCTGA }\end{array}$ & 190 & $39 \%$ & 557 & & 33.33 \\
\hline RPS $/ 8$ & $\begin{array}{l}\text { CTGCAGCCATGGT } \\
\text { AAGAGTT }\end{array}$ & $\begin{array}{l}\text { GTCTTCACGACAC } \\
\text { AACACGA }\end{array}$ & 350 & $38 \%$ & & & 33.35 \\
\hline RAB2L & $\begin{array}{l}\text { AAGTGTCATCANG } \\
\text { TCGTGTCC }\end{array}$ & $\begin{array}{l}\text { GCCCAGCGTAGCA } \\
\text { GTAGAG }\end{array}$ & 314 & $54 \%$ & 578 & 33.37 & \\
\hline
\end{tabular}

Markers in bold belong to the framework RH map, whereas markers in italic are positioned at the most likely location. Other markers have been developed in the frame of the contig building. The arrows on the side represent the fragments conserved between the pig and human genomes. Primer sequences, retention fraction, $\mathrm{RH}$ map location on porcine map and human location are indicated.

bT0079E18, bE0251O22, RPCI_440334P20 and bT220006) on the international BAC map (Humphrey et al, in preparation, and [17] that were overlapping with BAC already selected. To check for the accuracy of the global contig, new markers were developed using BES. The overlapping of BAC derived from the different INRA contigs was tested by PCR on BAC clones (Table 1 and Figure 2).

The building of the complete BAC contig was also monitored regularly by the mapping of BAC end- markers on the IMNpRH2 RH panel, verifying that the order of the markers and the distances between them were consistent with the BAC contig results. In particular, markers found at the extremities of the 11 INRA contigs were mapped on the RH panel. For these 19 markers, an average retention frequency of 35\% was observed. A 1000:1 framework map was then established, including previously mapped genes and 11 markers derived from BES. The 8 others BAC ends were mapped at their most likely location relatively to markers of the framework. This RH map covering $550 \mathrm{cR}$ is in total agreement with the $\mathrm{BAC}$ contig covering the whole region (Figure 2).

\section{Analysis of BAC contig}

The availability of BES from BAC located between PRIM2A (fragment 5) and GCLC (fragment 4) permitted characterization of the intermediate fragment in which no genes were mapped (Figure 2). All the significant homologies detected with these BES show that this fragment corresponds to fragment 6 located on HSA 6 in the interval 57.50 - 63.5 Mb framing the centromere. Among BES anchored at this human region, bI0666E01F is highly homologous to an intron of KHDRBS2, demonstrating the presence of the gene in this porcine region. In addition, the comparison of porcine BES produced from BAC bI0222C06, bI0223C10 and bI0819C05 revealed that these sequences share significant homology with 2 human fragments at a distance of $600 \mathrm{~Kb}$, suggesting the presence of duplication in the human genome. A dot-plot of the human region $57.73 \mathrm{Mb}-58.9 \mathrm{Mb}$ on itself was performed with Dotter, using its default settings [18]. The dot-plot showed that this region contains many large 


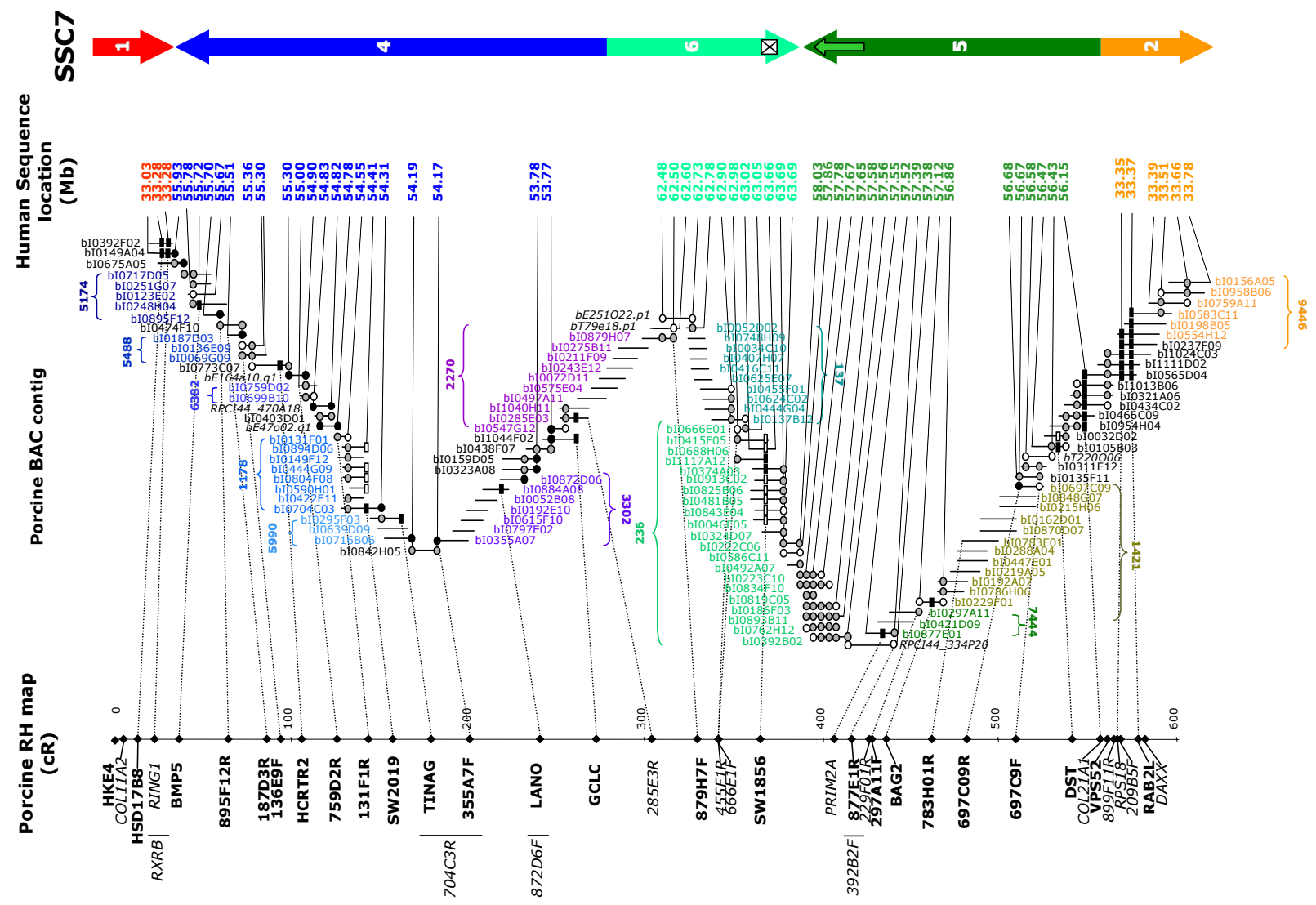

\section{Figure 2}

Porcine BAC contig with anchoring on porcine RH map and on human sequence after BAC end sequence analysis. The full contig is presented including mainly INRA BAC. Some additional BAC where recruited on the international analysis available at http://www.sanger.ac.uk/cgi-bin/Projects/S scrofa. The black circles represent sequenced BAC ends (BES) used for screening INRA BAC library. The white circles are BES for which a marker has been developed for a quality control of the contig by PCR on overlapping BAC (positive results are indicated with gray circles). The black boxes represent genes and microsatellites used for screening INRA BAC library, the white boxes indicate additional BAC on which a positive result has been obtained by PCR with these markers. Markers in bold on the RH map belong to the framework map, whereas markers in italic are additional markers mapped at the most likely location (when two locations are as likely, the marker is indicated on the left side of the map and its possible locations are indicated with a line). The BES for which a significant homology has been identified with sequence of HSA6 are illustrated by dotted lines. The large arrows represent the extent and the orientation of the different fragment conserved between pig and human genomes. The small arrows in fragment 5 represent the duplication identified on the human. Finally, the white box in fragment 6 is the fragment absent on the pig genome.

repeated sequences. A fragment of $290 \mathrm{~Kb}$ located at 57.74 $\mathrm{Mb}-58.03 \mathrm{Mb}$ is duplicated in position $58.34 \mathrm{Mb}-58.63$ $\mathrm{Mb}$, and a $50 \mathrm{~Kb}$ fragment from $58.07 \mathrm{Mb}$ to $58.12 \mathrm{Mb}$ is duplicated and turned over in position $58.73 \mathrm{Mb}-58.77$ Mb on HSA6 (results not shown). In the pig, this fragment covers $\sim 40 \mathrm{cR}$, which corresponds to $\sim 380 \mathrm{~Kb}$ suggesting that a single copy is present in the pig genome.
Finally, for BAC bI0688H06 and bI0046E05 mapped in the same region, hits obtained for the two BES of both clones were $650 \mathrm{~Kb}$ apart, which is not compatible with the size of a BAC. Analysis of this human region on ENSEMBL database [19] indicates that no porcine BES matches the human genome between 63.05 and 63.55 $\mathrm{Mb}$, whereas BES were anchored in the surrounding region on average each $20 \mathrm{~Kb}$. Altogether these results sug- 


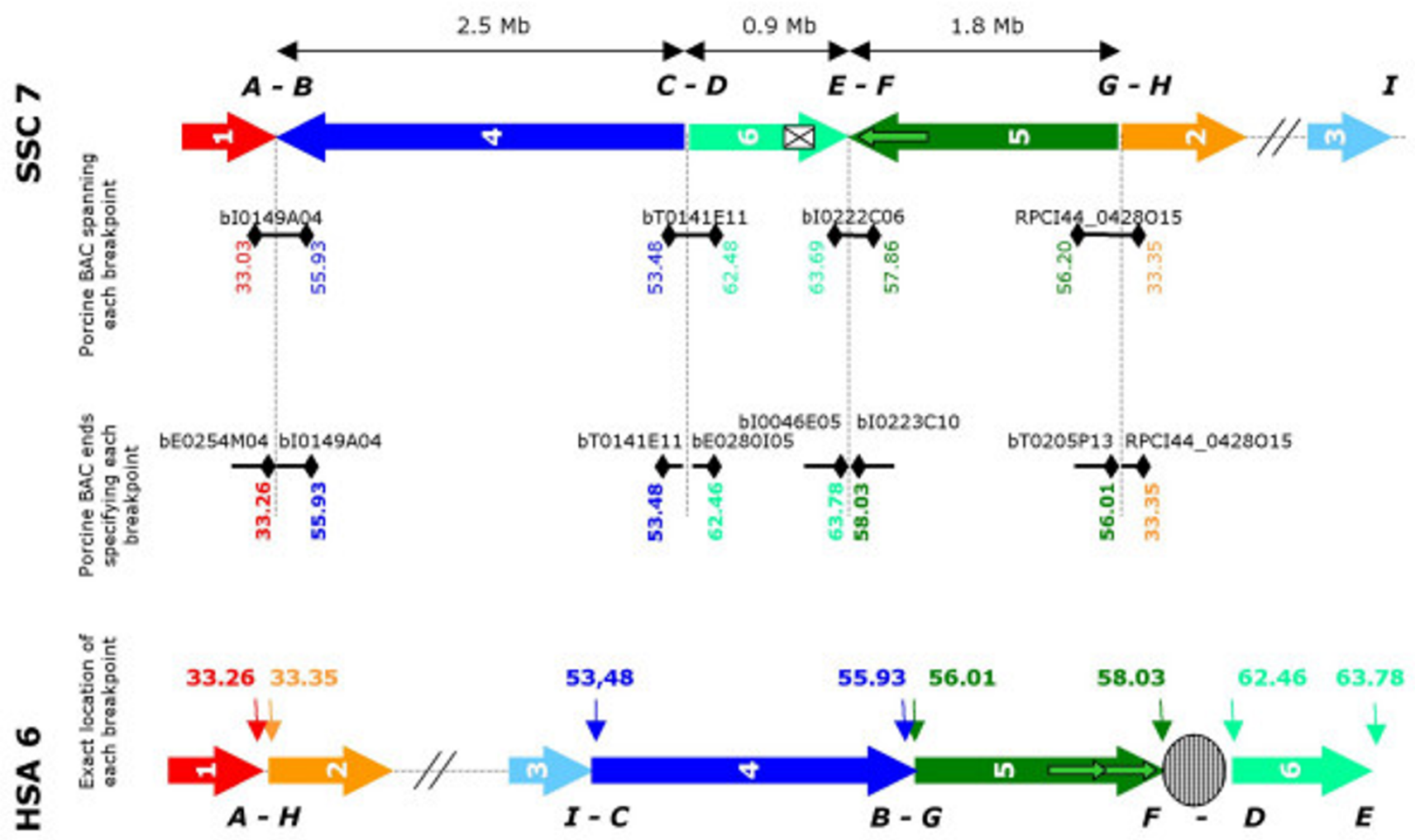

Figure 3

Description of the synteny breakpoints between SSC7 and HSA6. The arrows represent the various fragments of conserved synteny. The small green arrows symbolize a human duplication of a fragment of $290 \mathrm{~Kb}$. The white box represents a fragment of $500 \mathrm{~Kb}$ absent in the pig. Porcine BAC spanning each breakpoint are represented with anchoring of their ends on the human genome. Porcine BAC defining at best the localization of each breakpoint are shown with anchoring of their ends on the human genome.

gest that a $500 \mathrm{~Kb}$ region (in which no genes have already been identified on the human genome) is missing in the pig genome.

From this analysis, an estimate can be made of the size of the rearranged region on the pig genome - considering that the size of this region on the porcine genome is similar to the size of human region also found on the porcine genome. The sizes of the three rearranged fragments are: $\sim 2.5 \mathrm{Mb}$ for fragment $4,1.8 \mathrm{Mb}$ for fragment 5 and $0.9 \mathrm{Mb}$ for fragment 6 . The whole rearranged fragment covers thus $\sim 5.2 \mathrm{Mb}$ of the porcine genome.

To confirm the reality of the rearrangements identified during the building of the BAC contig, we tried to identify a porcine BAC spanning each of the 4 breakpoints, with BES anchored on the 2 expected locations on human genome (Figure 3). In order to increase the probability of identifying these $\mathrm{BAC}$, we also selected from the international map [17] BAC overlapping INRA BAC close to synteny breaking points. For BAC with no significant homology, we used FASTA to detect any potential homology, using less stringent parameters. In pig, one BAC anchored on the 2 expected human locations was identified, for the 4 breaking points A-B, C-D, E-F and G-H (bI0149A04 (33.03 Mb - 55.93 Mb), bT0141E11 (53.48 Mb-62.48 Mb), bI0222C06 (63.7 Mb - 57.85 Mb) and RPCI44_0428O15 (56.20 Mb - 33.35 Mb)) (Figure 3).

Analysis of the anchorage on the human map of all BES available in these regions, allowed us to estimate more precisely the location of the 4 breakpoints on the human genome (Figure 3): A/H breakpoint is located at 33.26 $33.35 \mathrm{Mb}, \mathrm{I} / \mathrm{C}$ at $53.24-53.48 \mathrm{Mb}, \mathrm{B} / \mathrm{G}$ at $55.93-56.01$, 


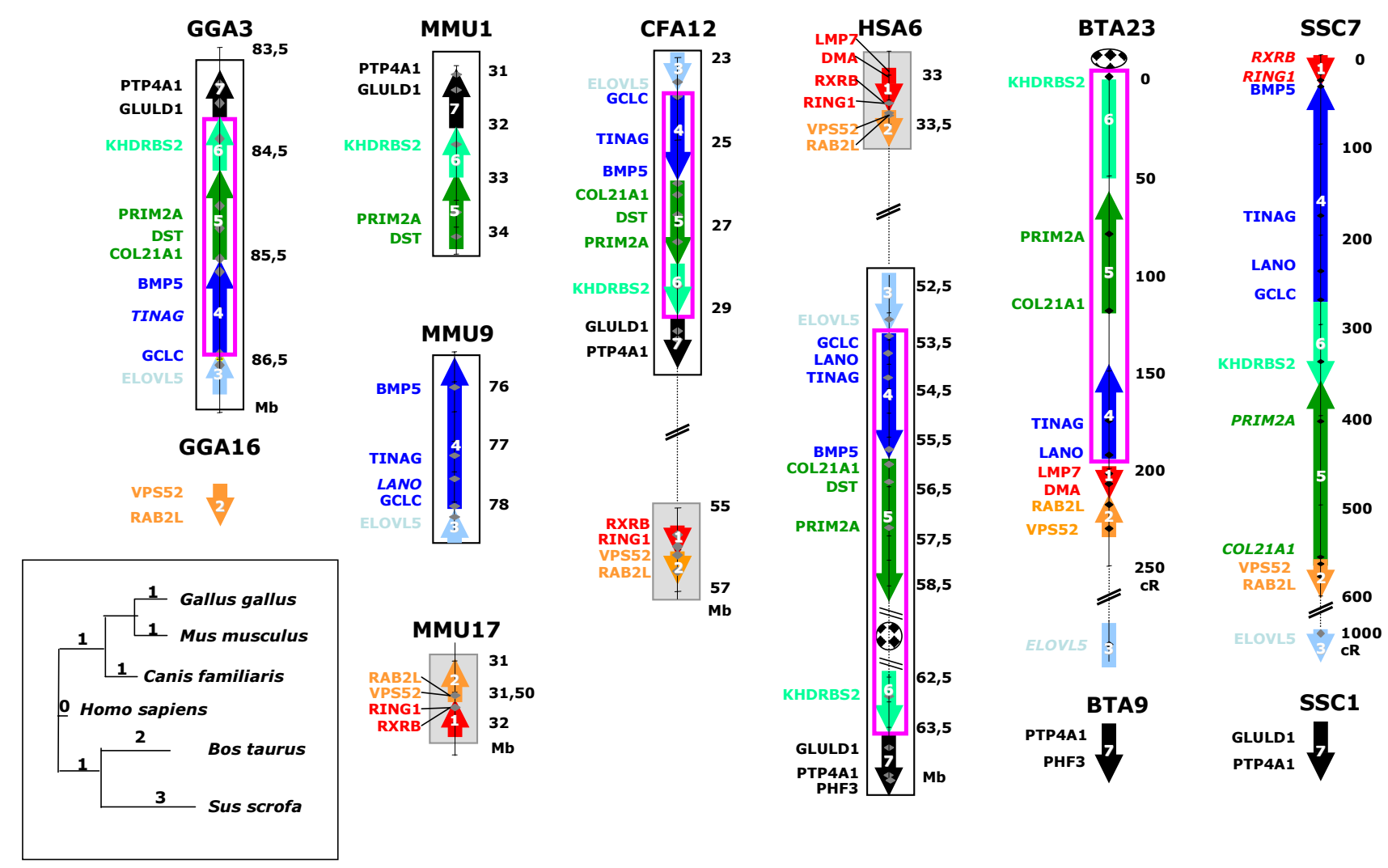

Figure 4

Multi-species (Gallus gallus, Sus scrofa, Bos taurus, Canis familiaris, Mus musculus and Homo sapiens) comparative map. Data concerning Canis familiaris, Gallus Gallus, Mus musculus, and Homo sapiens result respectively from Dog Jul. 2004 (canFam I) assembly, Chicken Feb. 2004 (galGal2) assembly, mouse Build 33 assembly by NCBI and human NCBI build 35. Data on chicken GGA 16 were not available on genome assembly, and were produced on ChickRH panel [20]. Maps concerning Sus scrofa and Bos taurus, are 1000:I framework maps produced on RH panel (see Figure 2). Markers written in bold belong to the framework map and markers in italic are mapped at their most likely location. The different arrows represent conserved synteny group. White boxes framed in black represent fragments conserved between Gallus gallus, Canis familiaris, Mus musculus and Homo sapiens. Boxes framed in pink represent fragments conserved between Gallus gallus, Canis familiaris, Mus musculus, Homo sapiens and Bos taurus. Grey boxes represent fragments conserved between Canis familiaris, Mus musculus and Homo sapiens.

$\mathrm{F} / \mathrm{D}$ after $58.03 \mathrm{Mb}$ and before $62.46 \mathrm{Mb}$ (with human centromere located in this interval), E point after 63.78 $\mathrm{Mb}$.

\section{Multispecies comparison of the organization of the studied region}

To compare the order of genes in this region between various species, we identified the location on the genome of sequenced species of several genes representing each chromosomal fragment described previously: fragment 1 (RXRb, RING1), fragment 2 (VPS52, RPS18 and RAB2L), fragment 3 (ELOVL5), fragment 4 (GCLC, LANO, TINAG and BMP5), fragment 5 (COL21A1, DST and PRIM2A), fragment 6 (KHDRBS2) and fragment 7 (GLULD1). Their locations were determined by blasting the porcine sequences against current genome assemblies: mouse sequence Build 33 assembly from NCBI, dog whole genome shotgun (WGS) assembly v1.0 (July 2004), and chicken galGal2 assembly. To enrich the set of data available for the chicken genome, we developed markers for VPS52 and RAB2L which were not identified on the sequence of chicken genome with the ESTs available for these genes. We mapped these 2 markers on chickRH panel [20] and analyzed the results on ChickRH server [21]. 
Table 2: Bovine markers developed for RH mapping

\begin{tabular}{|c|c|c|c|c|c|c|}
\hline STS-EST & SEQUENCE UP & SEQUENCE DN & $\begin{array}{l}\text { SIZE OF PCR } \\
\text { FRAGMENT }\end{array}$ & $\begin{array}{l}\text { \% RETENTION } \\
\text { ON RH5000 }\end{array}$ & $\begin{array}{l}\text { POSITION } \\
\text { (cR) }\end{array}$ & $\begin{array}{c}\text { HUMAN } \\
\text { LOCATION } \\
\text { (Mb) }\end{array}$ \\
\hline KHDRBS2 & $\begin{array}{l}\text { ATGGGGTTGCAAACAA } \\
\text { GAAG }\end{array}$ & $\begin{array}{l}\text { TTTGACAAATTGTGGTT } \\
\text { TCCT }\end{array}$ & 300 & 36 & 0 & 62.75 \\
\hline PRIM2A & $\begin{array}{l}\text { AAAGCAGCATTCTGGA } \\
\text { CTGG }\end{array}$ & $\begin{array}{l}\text { CCAGAAAAGGTCTGGG } \\
\text { TCAA }\end{array}$ & 104 & 40 & 82 & 57.29 \\
\hline BAG2 & $\begin{array}{l}\text { AATCTGACTGCGAACC } \\
\text { GTCT }\end{array}$ & $\begin{array}{l}\text { GTTTCCCAGGTCATCCA } \\
\text { GAA }\end{array}$ & 150 & 36 & 94 & 57.15 \\
\hline DST & $\begin{array}{l}\text { CCACCTGGACCTGAGA } \\
\text { CCTA }\end{array}$ & $\begin{array}{l}\text { ACAGGCGCTACTGTCC } \\
\text { ACTC }\end{array}$ & 101 & 39 & 106 & 56.58 \\
\hline COL2IAI & $\begin{array}{l}\text { CAGTTCTTCGGAGCCTT } \\
\text { GAG }\end{array}$ & $\begin{array}{l}\text { GACTGCAACTGACTGG } \\
\text { CTGA }\end{array}$ & 133 & 34 & 125 & 56.12 \\
\hline TINAG & $\begin{array}{l}\text { ACCGCATAGCAATTCAG } \\
\text { TCC }\end{array}$ & $\begin{array}{l}\text { ACCACCAAGCCCTATC } \\
\text { AACA }\end{array}$ & 123 & 31 & 184 & 54.28 \\
\hline LANO & $\begin{array}{l}\text { CGGTCTCCAGCTTGATT } \\
\text { GTT }\end{array}$ & $\begin{array}{l}\text { TCCAAGAGAAGGTGGG } \\
\text { TTTG }\end{array}$ & 185 & 37 & 202 & 53.77 \\
\hline RAB2L & $\begin{array}{l}\text { TCAATCTGGTCCCCTCT } \\
\text { CAG }\end{array}$ & $\begin{array}{l}\text { CCAGGACAGAGCTGAC } \\
\text { CACT }\end{array}$ & 141 & 33 & 230 & 33.37 \\
\hline B3GALT4 & $\begin{array}{l}\text { GGAGGCAAGCACCAGG } \\
\text { TAT }\end{array}$ & $\begin{array}{l}\text { CTGGCCACCTTCAGGA } \\
\text { TAAG }\end{array}$ & 125 & 37 & & 33.35 \\
\hline RINGI & $\begin{array}{l}\text { ATCCAAAGTGACCCCA } \\
\text { CAAG }\end{array}$ & $\begin{array}{l}\text { GGCAATAGAAAAGGCA } \\
\text { GCAG }\end{array}$ & 228 & 37 & & 33.29 \\
\hline HKE4 & $\begin{array}{l}\text { GACTATGTCCGTGGTG } \\
\text { CAAA }\end{array}$ & $\begin{array}{l}\text { CTGTACGAAGACCTGC } \\
\text { ACGA }\end{array}$ & 181 & 37 & & 33.28 \\
\hline$R \times R B$ & $\begin{array}{l}\text { CTGAAAAAGGTGGTGG } \\
\text { TGGT }\end{array}$ & $\begin{array}{l}\text { CATAAGAGCCGCAGAG } \\
\text { AACC }\end{array}$ & 193 & 37 & & 33.27 \\
\hline VPS52 & $\begin{array}{l}\text { ACCCAGCTGATCCAGC } \\
\text { TCTA }\end{array}$ & $\begin{array}{l}\text { ATCAGAAGTTGGGCTTG } \\
\text { TGC }\end{array}$ & 138 & 29 & & 33.33 \\
\hline ELOVL5 & $\begin{array}{l}\text { TCATGCTTTGATTTTGC } \\
\text { ACAT }\end{array}$ & $\begin{array}{l}\text { TGTAAACACGAAGCCG } \\
\text { TGACT }\end{array}$ & 104 & 28 & & 53.28 \\
\hline PTP4AI & $\begin{array}{l}\text { GTGATTCCCAGCCTCTC } \\
\text { TTG }\end{array}$ & $\begin{array}{l}\text { CCACTTGTTCCCGGAC } \\
\text { AGTA }\end{array}$ & 199 & 50 & & 64.35 \\
\hline PHF3 & $\begin{array}{l}\text { CCATTTCGAAGAGGATC } \\
\text { AGC }\end{array}$ & $\begin{array}{l}\text { AACCCAGGTGGGCTAG } \\
\text { ACTT }\end{array}$ & 188 & 37 & & 64.45 \\
\hline
\end{tabular}

Markers in bold belong to the framework RH map whereas markers in italic are positionned with the best likelihood. Primer sequences, retention fraction, $\mathrm{RH}$ map location on bovine map and human location are indicated.

It was also important to include a species phylogenetically close to the pig in the comparison. We selected cattle, a species for which both detailed comparative mapping studies have been performed [22,23] and a partial genomic sequence is available. However, in the region of interest, the assembly of the bovine genome is by no means complete (each gene is found in a different scaffold after blasting the sequence of genes mapped in pig against the bovine genome) and available RH maps are insufficiently detailed. This led us to develop a bovine RH map in this region. We used the ICCARE browser [13], to select bovine EST available for 16 genes of this region: $R X R b$, RING1, HKE4, HSD17B8, VPS52, RAB2L, ELOVL5, TINAG, LANO, COL21A1, DST, BAG2, PRIM2A, KHDRBS2, PHF3 and PTP4A1 (Table 2). We developed pairs of primers for each gene and we mapped them on the RH5000 bovine panel [22]. An average retention frequency of $36 \%$ was observed, ranging from $26 \%$ for GLO1 to $50 \%$ for PTP4A1. Vectors obtained for the 16 new genes were analyzed together with 31 markers previ- ously available for BTA23 [22]. A 1000:1 framework map including 10 genes, was built with Carthagene software [14] in the region from PRIM2A to VPS52 (Figure 1B). In addition gene KHDRBS2 is linked with a LOD score 3.5 at the top of this map. Four additional genes HSD17B8, $R X R b$, RING1, and HKE4 were found totally linked and mapped at their most likely location relatively to markers of the framework (Figure 1B). ELOVL5 was found linked to markers previously mapped in a distal position. PHF3 and PTP4A1 were found significantly linked together $(\mathrm{LOD}=13.2)$, but totally unlinked to other genes.

In the dog genome, all the selected genes are grouped into two large fragments (fragments 3, 4, 5, 6 and 7 and fragments 1 and 2) located on chromosome 12 (Figure 4). In the mouse genome, the same region is similarly split into 3 conserved fragments (Figure 4): fragments 5, 6 and 7 are located on MMU1; fragments 3 and 4 on MMU9; and fragments 1 and 2 on MMU17. In chicken, a conserved fragment containing fragments $3,4,5,6$ and 7 is located on 
GGA3. Two other genes, VPS52 and RAB2L (fragment 2), are mapped in a linkage group that also contains markers derived from chicken MHC region located on GGA16 (Figure 4). No information is available for $R X R B$ and RING1 (fragment 1). If the order of genes is thus globally conserved in these species, a very different situation is observed on the bovine genome (Figure 4): 14 of the 16 selected genes are localized on BTA23, but in an order very different from the generally conserved order, and 2 others genes (PHF3 and PTP4A1 representing fragment 7) are located on chromosome 9.

The analysis of these results shows that the whole fragment (comprising fragments 4, 5 and 6) inserted on the pig genome between fragments 1 and 2 (RING1 and VPS52) seems very well conserved in chicken, dog, mouse and human. In cattle and pig, belonging both to the clade of Cetartiodactyles, fragments 4, 5 and 6 are found at a different location. However, in this $5.2 \mathrm{Mb}$ large fragment, a total conservation of gene order was observed between human and cattle genomes. A global analysis of the chromosomal rearrangements that occurred during evolution was carried out using GRIMM. [24]. The results obtained are summarized on Figure 4.

\section{Discussion}

The study of porcine chromosome 7 is of particular interest due to the presence of many QTL affecting traits of economic importance and of MHC $[1,2]$. One approach to study this chromosome is to identify all the genes located on this chromosome and to compare their order relatively to that in the human genome. Previously, a strong conservation of gene order was found between a large fragment of porcine SSC7 and human HSA6 p arm [9,10]. We had highlighted the existence of a fragment estimated to be less than $4 \mathrm{Mb}$ rearranged between SSC7 and HSA6 [11]. This fragment defined by 3 genes (LANO previously named FLJ10775, BMP5 and BAG2) located on HSA6p12.1-6p11.2 is found in the pig genome between genes located on HSA6p21.32 [11]. The building of a BAC contig in the chromosomal region surrounding SLA complex provided additional information, even if the contig remained incomplete [12]. This study describes a situation that is even more complex, suggesting the presence of VPS52 (previously named SACM2L) within the rearranged region. In the pig genome, this rearranged chromosomal region is included in the region where the QTL of interest is mapped, between markers Sw1856 and NFY (Demeure et al, JAS, in press). In order to identify all candidate genes that may be responsible for the QTL effects, it was particularly important to establish a detailed comparative mapping analysis of this region.

We first built a dense porcine RH map (Figure 1A) using the high resolution IMNpRH2 12000 rad panel, allowing us to order unambiguously genes at distances of 100 to 600 $\mathrm{Kb}$ [7]. Our data confirmed the shift of the rearranged fragment and its precise location in the pig genome between RING1 and VPS52 genes (previously called $S A C M 2 L$ ) (fragments 1 and 2). In contrast to Barbosa et al [12], we show that VPS52 is located close to RAB2L in both species. We also determined more precisely the extent of the fragment rearranged between the human and pig genomes. The upper frontier of this fragment is located between ELOVL5 (previously named HELO1) and GCLC, located respectively at 53.28 and $53.43 \mathrm{Mb}$ on HSA6. The lower boundary of the fragment is defined by the first gene located on HSA6q also mapped on SSC1. This first gene was previously designated as FLJ13159, and was located at $71.53 \mathrm{Mb}$ on HSA6 [11]. We showed that GLULD1, located at $64.05 \mathrm{Mb}$ on HSA6, also mapped on SSC1 and constitutes the limit currently identified in this fragment.

The gene-based RH map (Figure 1A) that we built allowed us to define 3 sub-fragments of this region arranged differently on the pig and human genomes. Two of these subfragments are delimited by genes: BMP5 and GCLC for the first one (fragment 4), and PRIM2A and COL21A1 for the second (fragment 5). No genes were identified in the third fragment (fragment 6), which contains the anonymous microsatellite Sw1856. This map greatly refines the internal rearrangements suggested by our previous work [11]. The sum of the sizes of fragments 4 and 5 is $\sim 4.3 \mathrm{Mb}$ and is similar to the orthologous human region. Fragment 6 covers $1.4 \mathrm{Mb}$ in the human map, whereas the size of this fragment in the porcine RH map suggests a size of approximately $900 \mathrm{~Kb}$ which would indicate that a fragment of $500 \mathrm{~Kb}$ is absent in the porcine genome. The fragment containing Sw1856 on the pig genome might thus be orthologous to part of the region flanking the centromere of HSA6, but at that step it remained to be proved (Figure 1A).

To confirm the order of genes suggested by RH mapping and study this region in more detail, we built a contig of BAC covering the entire region (Figure 2 ). The availability of elementary contigs resulting from the fingerprinting of clones from the INRA BAC library and sequences of extremities of 35000 of these BAC (BES) (Chardon et al, in preparation) greatly facilitated the establishment of this complete contig. Our work completes the studies begun by Barbosa et al [12], who presented 4 small contigs. The analysis of porcine BES anchoring on the human genome demonstrates that the fragment 6 containing porcine marker Sw1856 corresponds to a fragment framing the centromere of HSA6 (6p11.1-6q11.1). Taking into account the average distance between two hits of porcine BES on human genome, we can say that if additional fragments from other human chromosomes are located in 
this rearranged region of the porcine genome, they should measure $<50 \mathrm{~Kb}$.

Analysis of the anchoring of porcine BES on the human genome highlighted also additional results: 1) It was indeed possible to define the position of the 3 synteny breakpoints (Figure 3). On the human genome, these synteny breakpoints (A-H, I-C and B-G) are situated between genes RING1-VPS52, ELOVL5-GCLC and BMP5COL21A1. Each of these 3 synteny breakpoints are localized in a interval of $\sim 100-200 \mathrm{~Kb}$, between the human positions $33.26 \mathrm{Mb}-33.35 \mathrm{Mb}, 53.24 \mathrm{Mb}-53.48 \mathrm{Mb}$ and $55.93 \mathrm{Mb}-56.01 \mathrm{Mb}$. 2) The fourth synteny breakpoint (E$\mathrm{F}$ on the pig genome and F-D on the human genome) was identified thanks to the anchoring of the BES in the human genome: the two fragments framing HSA6 centromere were found in a different order on the pig genome without the presence of a centromere (Figure 3). 3) For several BES, alignment on the human genome showed 2 possible positions with similar likelihood. This result suggested the existence of duplicated sequences close to the human centromere, which was confirmed by a dot plot on itself of the sequence of this human region. Phylogeny studies of human chromosome 6 demonstrated the repositioning of this centromere among primate species in a region equivalent to 6p22.1 in Eulemur macaco, Callithrix jacchus and Lagothrix lagothrica, to 6q22 in Macaca fascicularis and Presbytis cristata, and to 6p12 in the great apes [25]. The repeated sequences we highlighted might then correspond to sequences of an ancestral centromere positioned in HSA6p22.1 [25]. 4) Lastly, in silico analysis of the BES alignments on the human genome provided evidence of the absence from the pig genome of a fragment of $\sim 400-500 \mathrm{~Kb}$ found in human genome. One can argue that the 2 BES of BAC bI0688H06 match on the human genome with sequences distant of $650 \mathrm{~Kb}($ at $63.02 \mathrm{Mb}$ and $63.67 \mathrm{Mb}$ ). The absence of anchoring of other BES between $63.05 \mathrm{Mb}$ and $63.55 \mathrm{Mb}$, whereas one hit is found for each $20 \mathrm{~Kb}$ in the surrounding region, confirmed that this fragment is absent from the pig genome.

This region appears to be heavily rearranged between the porcine and human genomes. We wanted to determine if these rearrangements are specific of suines or Cetartiodactyles, or occur in many other species. To identify gene order in other species, we used the genomic sequence when available. For Gallus gallus, the assembly of the genome has not been totally completed; this is why we enriched information obtained from the sequence by $\mathrm{RH}$ mapping data when it was necessary (Figure 4). The order of genes seems to be conserved between the chicken and human genomes except for the $R X R b$ and RING1 genes (belonging to fragment 1 ). These 2 genes which may not exist in the chicken genome are located in other species in the extended class II region of MHC. The chicken MHC, named the B locus, is a $92 \mathrm{~Kb}$ DNA sequence containing 19 genes [26,27]. It defines a minimal essential set of MHC genes conserved over 200 million years of divergence between birds and mammals [27].

As the available assembly of the cow genome consists of many independent sequence scaffolds, we determined the gene order by RH mapping [22] (Figure 1B). Our results reveal that genes from GCLC to KHDRBS2 (fragments 4, 5 and 6) are similarly ordered on the human and bovine genomes in this region, which suggests that the internal rearrangements of these 3 fragments, comparing the human and porcine genomes, are specific to the evolution of the suine family. Everts-van der Wind et al. [18] reported that 12 cattle chromosomes are entirely similar to a complete human chromosome arm, and in particular, that $\mathrm{p}$ and $\mathrm{q}$ arms of HSA6 are homologs of BTA23 and BTA9. Our study modifies slightly this report, in that KHDRBS2 (belonging to fragment 6), the first gene localized on the long arm of human chromosome 6 , is probably located on bovine chromosome 23. As the number of genes in this region, as in other species, is very limited; it is difficult to develop and map markers in this region. The end of BTA23 might thus signal the location of the centromere in the ancestors of Cetartiodactyles, in which the fission between the 2 chromosomal arms occurred. In this region, a further comparison of the bovine genomic sequence with the human sequence will be of interest.

We compared the gene order in the pig (Sus scrofa) and cattle (Bos Taurus) with the situation in the genomic sequence of Mus musculus, Canis familiaris, Gallus gallus and Homo sapiens (Figure 4). We observed at first that on the pig genome, a large fragment (comprising fragments 4,5 and 6) of $~ 5.2 \mathrm{Mb}$ is shifted between RING1 and VPS52, 2 genes belonging to the extended class II region of the MHC. If MHC is one of the most dynamic regions of the genome [28], a high level of conservation of genome organization provides evidence in this region of extended class II genes [29]. Fourteen genes occur in this region between RING1 and the VPS52 in dog, cat, human and mouse genomes, which only differs in the presence of processed pseudogenes [29]. This small fragment of 500 $\mathrm{Kb}$ containing RING1 and VPS52 is thus dynamically involved in chromosomal evolution. It is interesting to note that the translocation of the $5.2 \mathrm{Mb}$ fragment occurs in this region. In this region, the genomic organization of the fragment and its flanking regions is similar in mouse, human, dog and chicken, whereas translocation is observed in pig and cattle. This suggests that this genomic reorganization occurred after the divergence of the common ancestor of Cetartiodactyles from the Ferungulates ancestor between 94 to 62 million years ago [30]. The gene order observed in pig for the $5.2 \mathrm{Mb}$ fragment is different from the one identified in other species, indicating 
that these internal rearrangements occurred after ruminant-suine speciation. We identified a situation really different from the map presented by Everts-van der Wind et al. for the proximal part of BTA23 close to the centromere [23]. Further study of the rearrangements that occurred in ruminants in this region of extended class II of the MHC complex is clearly needed.

We used GRIMM to compare the different maps available and to present a possible evolution of this region between the various species [24]. The unrooted tree we obtained (Figure 4) presents a very different view from a classical phylogenic tree $[31,32]$, highlighting a high rate of rearrangements in this region that have occurred recently in Cetartiodactyles. The study of local rearrangements also provides a view different from the recent multispecies comparison of this region [33]. In order to filter out noise induced by mapping errors, this analysis from Murphy et al only took into account fragments $>3 \mathrm{Mb}$, defined by three genes. Among other results, they documented the reuse of evolutionary breakpoint regions identified between conserved homologous synteny blocks. Our study indicates that such a study should be redone on a more detailed scale, when the accuracy of mapping data is adequate.

\section{Conclusion}

A QTL affecting fattening and growth had been mapped in the porcine region SSC $7 \mathrm{q} 1.2$. As we had shown that a small fragment is translocated between porcine and human genome, it was important to establish a final comparative map of this region. The RH map and the BAC contig we developed in this region allowed us to attest that the translocated fragment is approximately $5.2 \mathrm{Mb}$ long. It consists in three blocks, found in HSA6 between 53.45 and $63.5 \mathrm{Mb}$, one of these blocks containing a few genes, framing the centromere on the human chromosome. The multispecies study allowed us to confirm that the translocation of this $5.2 \mathrm{Mb}$ fragment occurred in the common ancestor of pig and ruminants, whereas internal rearrangements are found only in the pig genome. The exact knowledge of the genomic content of this region permits us now to establish a list of all possible candidate genes that may affect the growth and fattening of pigs.

\section{Methods}

\section{Markers and PCR amplification}

The ICCARE (Interspecific Comparative Clustering and Annotation foR Ests) tool available at [13] allowed us to select 16 genes in the region between RXRB and RAB2L, for which a porcine EST sequence is available (Table 1 ). In addition, 88 markers have been developed from INRA BAC ends. Several result from personal sequencing using the BigDye Terminator V3.1 cycle sequencing kit (Applied Biosystems) on the ABI 3700 automatic sequencer; these sequences were submitted to EMBL Nucleotide Sequence Database (AM156864 to AM156903) [34]. Others come from BAC ends sequences available on ENSEMBL [19]. Primers have been chosen using Primer3 software [35]. PCR was performed in a $15 \mu \mathrm{l}$ reaction volume containing $25 \mathrm{ng}$ template DNA, $200 \mu \mathrm{M}$ dNTP, $0.25 \mu \mathrm{M}$ specific primer pair and 0.5 U Taq polymerase (GoTAQ) in the reaction buffer supplied by the manufacturer. Amplifications were carried out on a Gene-Amp System 9700 (Applied Biosystems) thermocycler. Thermal cycling parameters were defined as follows: denaturation at $94^{\circ} \mathrm{C}$ for $5 \mathrm{~min}$, followed by 32 cycles of (1) $94^{\circ} \mathrm{C}$ for $45 \mathrm{sec}$, (2) annealing temperature for $45 \mathrm{sec},(3) 72^{\circ} \mathrm{C}$ for $45 \mathrm{sec}$, and a final extension was performed at $72^{\circ} \mathrm{C}$ for $5 \mathrm{~min}$. PCR products were analyzed on a $2 \%$ agarose gel, and visualized after ethidium bromide staining.

\section{RH mapping}

Markers were mapped on the IMpRH panel [6] and IMNpRH2 panel [7] according to the INRA protocols. For markers used on IMpRH panel, vectors were submitted to the IMpRH web server at [36] for an initial two-point assignment. Then, vectors obtained on IMpRH and IMNpRH2 were analyzed with Carthagene software [14]. A 1000:1 framework map was built with buildfw option using a stepwise locus adding strategy under an haploid model of fragment retention. The different provisional frameworks were checked using the simulated annealing algorithm to test inversion of the map fragments, and the flips algorithm to test all local permutations in a window of 6 markers. The resulting RH map was drawn with Mapchart 2.0 [37]. An RH map was also established for the bovine genome using the bovine RH panel RH5000 [22]. Several markers were also mapped on the chicken genome using ChickRH6 panel [20].

\section{$B A C$ screening and chromosome walking}

The INRA BAC porcine library [12], was screened by PCR on BAC super pools and pools. The presence of the expected sequence in the identified BAC was checked by PCR on DNA from the isolated BAC clone. For the selected BAC that had been previously fingerprinted and incorporated in a primary INRA contig (Chardon et al in preparation), all BAC from this contig were selected from INRA BAC Server [38]. To confirm the BAC overlapping, markers developed from BES were tested against DNA of $\mathrm{BAC}$ belonging to its primary contig or BAC located at the extremities of adjacent contigs.

\section{Multispecies comparison}

In order to determine genes order in other species, 12 genes (RXRB, RING1, VPS52, RAB2L, ELOVL5, GCLC, BMP5, COL21A1，DST, PRIM2A，KHDBRS2, GLULD1) were located with Blast in human by using NCBI build 35 produced by the International Human Genome Sequenc- 
ing Consortium, in mouse (Mus musculus) draft genome data was obtained from the Build 33 assembly by NCBI, in chicken (Gallus gallus) draft assembly was produced by the Genome Sequencing Center at the Washington University School of Medicine in St. Louis, in dog (Canis familiaris) whole genome shotgun (WGS) assembly v1.0 was sequenced and assembled by the Broad Institute of MIT/Harvard and Agencourt Bioscience. We used the GRIMM software [24] to estimate the rearrangements between species and build a multispecies phylogenetic tree.

\section{List of abbreviations}

MHC major histocompatibility complex; QTL quantitative trait locus; SLA swine leucocyte antigen; RH radiation hybrid; BAC bacterial artificial chromosome; BES BAC end sequence, $\mathrm{cR}$ centi Ray; HSA human chromosome; SSC porcine chromosome; MMU murine chromosome; BTA bovine chromosome; GGA chicken chromosome; CFA dog chromosome; IMNpRH2 INRA Minnesota Nevada porcine Radiation Hybrid panel 2.

BAC bIxxxZxx are from INRA BAC library [12], BAC bExxxZxx are from CHORI242 library (P. de Jong, et al., unpubliched), BAC bTxxxZxx are from Roslin BAC library [39], RPCI44_xxxZxx are BAc clones from RPCI44 library (P. de Jong et al., unpublished).

\section{Authors' contributions}

JD built the complete contig using the INRA BAC library fingerprinting, did the genotyping of the porcine and bovine RH panels, made the in silico analyses and drafted the manuscript. JR supervised this work. KF took part in the development and the explanation of the techniques. MG provided DNA of bovine RH5000 panel and the vectors for the bovine markers previously mapped in the studied region. MM did the genotyping and the analyses on the chicken RH panel. CR performed some preliminary BAC screening. PC coordinated the fingerprinting of INRA $\mathrm{BAC}$ and the providing of all BAC necessary to the construction of the contig. DM conceptualized the investigation, analyzed the RH data, and finalized the manuscript.

\section{Acknowledgements}

We would like thank Laurent Schibler, Anne Roig and Jean Claude Save for providing the fingerprints of BAC clones of INRA library, F. Puimi, and the CRB GADIE (Jouy en Josas, France) for providing the BAC clones. We also thank the CNS (Evry, France) for the BAC ends sequencing, and T. Faraut and $\mathrm{P}$. Dehais for help in sequence comparison. JD is supported by a PhD grant from the FSE (Fonds Social Européen).

\section{References}

I. Milan D, Bidanel JP, lannuccelli N, Riquet J, Amigues Y, Gruand J, Le Roy P, Renard C, Chevalet C: Detection of quantitative trait loci for carcass composition traits in pigs. Genet Sel Evol 2002, 34:705-728.

2. Bidanel JP, Milan D, lannuccelli N, Amigues $Y$, Boscher MY, Bourgeois F, Caritez JC, Gruand J, Le Roy P, Lagant H, Quintanilla R, Renard C,
Gellin J, Ollivier L, Chevalet C: Detection of quantitative trait loci for growth and fatness in pigs. Genet Sel Evol 200I, 33:289-309.

3. Wada $Y$, Akita $T$, Awata $T$, Furukawa $T$, Sugai $N$, Inage $Y$, Ishii $K$, Ito Y, Kobayashi E, Kusumoto H, Matsumoto T, Mikawa S, Miyake M, Murase A, Shimanuki S, Sugiyama T, Uchida Y, Yanai S, Yasue H: Quantitative trait loci (QTL) analysis in a Meishan x Gottingen cross population. Anim Genet 2000, 31 : 376-384.

4. Rattink AP, De Koning DJ, Faivre M, Harlizius B, van Arendonk JA, Groenen MA: Fine mapping and imprinting analysis for fatness trait QTLs in pigs. Mamm Genome 2000, I 1:656-66I.

5. Malek M, Dekkers JC, Lee HK, Baas TJ, Prusa K, Huff-Lonergan E, Rothschild MF: A molecular genome scan analysis to identify chromosomal regions influencing economic traits in the pig. II. Meat and muscle composition. Mamm Genome 200I, I 2:637-645

6. Yerle M, Pinton P, Robic A, Alfonso A, Palvadeau $Y$, Delcros C, Hawken R, Alexander L, Beattie C, Schook L, Milan D, Gellin J: Construction of a whole-genome radiation hybrid panel for highresolution gene mapping in pigs. Cytogenet Cell Genet 1998, 82:182-188.

7. Yerle M, Pinton P, Delcros C, Arnal N, Milan D, Robic A: Generation and characterization of a 12,000-rad radiation hybrid panel for fine mapping in pig. Cytogenet Genome Res 2002, 97:219-228.

8. Martins-Wess F, Milan D, Drogemuller C, Vobeta-Nemitz R, Brenig $B$, Robic A, Yerle M, Leeb T: A high resolution physical and RH map of pig chromosome 6q1.2 and comparative analysis with human chromosome 19q13.1. BMC Genomics 2003, 4:20.

9. Genet C, Renard C, Cabau C, Rogel-Gaillard C, Gellin J, Milan D: In the QTL region surrounding porcine MHC, gene order is conserved with human genome. Mamm Genome 200I, I 2:246-249.

10. Tanaka M, Matsumoto T, Yanai S, Domukai M, Toki D, Hayashi T, Kiuchi S, Yasue H, Uenishi H, Kobayashi E, Awata T: Conservation of the syntenies between porcine chromosome 7 and human chromosomes 6,14 and 15 demonstrated by radiation hybrid mapping and linkage analysis. Anim Genet 2003, 34:255-263.

II. Demeure O, Renard C, Yerle M, Faraut T, Riquet J, Robic A, Schiex $T$, Rink $A$, Milan $D$ : Rearranged gene order between pig and human in a QTL region on SSC 7. Mamm Genome 2003, | 4:71-80.

12. Barbosa A, Demeure O, Urien C, Milan D, Chardon P, Renard C: A physical map of large segments of pig chromosome 7qIIq14: comparative analysis with human chromosome 6p2l. Mamm Genome 2004, 15:982-995.

13. Muller C, Denis M, Gentzbittel L, Faraut T: The Iccare web server: an attempt to merge sequence and mapping information for plant and animal species. Nucleic Acids Res 2004, 32:W429-34.

14. de Givry S, Bouchez M, Chabrier P, Milan D, Schiex T: CARHTA GENE: multipopulation integrated genetic and radiation hybrid mapping. Bioinformatics 2005, 21:1703-1704.

15. UCSC GB: . [http://genome.ucsc.edu].

16. Rogel-Gaillard C, Bourgeaux N, Billault A, Vaiman M, Chardon P: Construction of a swine BAC library: application to the characterization and mapping of porcine type $C$ endoviral elements. Cytogenet Cell Genet 1999, 85:205-2II.

17. SANGER I: . [http://www.sanger.ac.uk].

18. Sonnhammer EL, Durbin R: A dot-matrix program with dynamic threshold control suited for genomic DNA and protein sequence analysis. Gene 1995, 167:GCI-10.

19. ENSEMBL D: http://www.ensembl.org.

20. Morisson M, Lemiere A, Bosc S, Galan M, Plisson-Petit F, Pinton P, Delcros C, Feve K, Pitel F, Fillon V, Yerle M, Vignal A: ChickRH6: a chicken whole-genome radiation hybrid panel. Genet Sel Evol 2002, 34:52I-533.

21. ChickRH WS: . [http://www.toulouse.inra.fr/lgc/chickrh/].

22. Williams JL, Eggen A, Ferretti L, Farr CJ, Gautier M, Amati G, Ball G, Caramorr T, Critcher R, Costa S, Hextall P, Hills D, Jeulin A, Kiguwa SL, Ross O, Smith AL, Saunier K, Urquhart B, Waddington D: A bovine whole-genome radiation hybrid panel and outline map. Mamm Genome 2002, 13:469-474.

23. Everts-van der Wind A, Kata SR, Band MR, Rebeiz M, Larkin DM, Everts RE, Green CA, Liu L, Natarajan S, Goldammer T, Lee JH, McKay S, Womack JE, Lewin HA: A 1463 gene cattle-human 
comparative map with anchor points defined by human genome sequence coordinates. Genome Res 2004, | 4: | 424- | 437.

24. Tesler G: GRIMM: genome rearrangements web server. Bioinformatics 2002, I 8:492-493.

25. Eder V, Ventura M, lanigro M, Teti M, Rocchi M, Archidiacono N: Chromosome 6 phylogeny in primates and centromere repositioning. Mol Biol Evol 2003, 20:1506-15I2.

26. Guillemot F, Billault A, Pourquie O, Behar G, Chausse AM, Zoorob R, Kreibich G, Auffray C: A molecular map of the chicken major histocompatibility complex: the class II beta genes are closely linked to the class I genes and the nucleolar organizer. Embo J 1988, 7:2775-2785.

27. Kaufman J, Milne S, Gobel TW, Walker BA, Jacob JP, Auffray C, Zoorob R, Beck S: The chicken B locus is a minimal essential major histocompatibility complex. Nature 1999, 40 I:923-925.

28. Kelley J, Walter L, Trowsdale J: Comparative genomics of major histocompatibility complexes. Immunogenetics 2005, 56:683-695.

29. Debenham SL, Hart EA, Ashurst JL, Howe KL, Quail MA, Ollier WE, Binns MM: Genomic sequence of the class II region of the canine MHC: comparison with the MHC of other mammalian species. Genomics 2005, 85:48-59.

30. Springer MS, Murphy WJ, Eizirik E, O'Brien SJ: Placental mammal diversification and the Cretaceous-Tertiary boundary. Proc Natl Acad Sci U S A 2003, I 00:1056-106I.

31. Bourque G, Pevzner PA, Tesler G: Reconstructing the genomic architecture of ancestral mammals: lessons from human, mouse, and rat genomes. Genome Res 2004, I4:507-5I6.

32. Bourque G, Pevzner PA: Genome-scale evolution: reconstructing gene orders in the ancestral species. Genome Res 2002, 1 2:26-36.

33. Murphy WJ, Bourque G, Tesler G, Pevzner P, O'Brien SJ: Reconstructing the genomic architecture of mammalian ancestors using multispecies comparative maps. Hum Genomics 2003, I:30-40.

34. EMBL NSD: . [http://www.ebi.ac.uk/embl/].

35. Primer3 S: . [http://frodo.wi.mit.edu/cgi-bin/primer3/ primer3 www.cgi].

36. Milan D, Hawken R, Cabau C, Leroux S, Genet C, Lahbib Y, Tosser G, Robic A, Hatey F, Alexander L, Beattie C, Schook L, Yerle M, Gellin : IMpRH server: an RH mapping server available on the Web. Bioinformatics 2000, I 6:558-559.

37. Voorrips RE: MapChart: software for the graphical presentation of linkage maps and QTLs. J Hered 2002, 93:77-78.

38. Server INRABACW: http://imprh.toulouse.inra.fr.

39. Anderson SI, Lopez-Corrales NL, Gorick B, Archibald AL: A largefragment porcine genomic library resource in a BAC vector. Mamm Genome 2000, I I:8I I-8I4.

\section{Publish with Bio Med Central and every scientist can read your work free of charge}

"BioMed Central will be the most significant development for disseminating the results of biomedical research in our lifetime. "

Sir Paul Nurse, Cancer Research UK

Your research papers will be:

- available free of charge to the entire biomedical community

- peer reviewed and published immediately upon acceptance

- cited in PubMed and archived on PubMed Central

- yours - you keep the copyright 